\title{
A Long-Period Totally Eclipsing Binary Star at the Turnoff of the Open Cluster NGC 6819 Discovered with Kepler ${ }^{1}$
}

\author{
Eric L. Sandquist ${ }^{2}$; Robert D. Mathieu ${ }^{3}$; Karsten Brogaard ${ }^{4,5}$; Soren Meibom ${ }^{6}$; Aaron M. \\ Geller $^{3,7}$; Jerome A. Orosz ${ }^{2}$; Katelyn E. Milliman ${ }^{3}$; Mark W. Jeffries, Jr. ${ }^{2}$; Lauren N. \\ Brewer $^{2}$; Imants Platais ${ }^{8}$; Frank Grundahl ${ }^{5}$; Hans Bruntt ${ }^{5}$; Soeren Frandsen ${ }^{5}$; Dennis \\ Stello $^{9}$
}

\begin{abstract}
We present the discovery of the totally eclipsing long-period $(P=771.8 \mathrm{~d})$ binary system WOCS 23009 in the old open cluster NGC 6819 that contains both an evolved star near central hydrogen exhaustion and a low-mass $\left(0.45 M_{\odot}\right)$ star. This system was previously known to be a single-lined spectroscopic binary, but the discovery of an eclipse near apastron using data from the Kepler space telescope makes it clear that the system has an inclination that is very close to $90^{\circ}$. Although the secondary star has not been identified in spectra, the mass of the primary star can be constrained using other eclipsing binaries in the cluster. The combination of total eclipses and a mass constraint for the primary star allows us
\end{abstract}

\footnotetext{
${ }^{2}$ San Diego State University, Department of Astronomy, San Diego, CA, 92182; erics@mintaka.sdsu.edu; orosz@sciences.sdsu.edu; jeffries@sciences.sdsu.edu; ezereve@gmail.com

${ }^{3}$ University of Wisconsin-Madison, Department of Astronomy, Madison, WI, 53706; mathieu@astro.wisc.edu; milliman@astro.wisc.edu

${ }^{4}$ Department of Physics \& Astronomy, University of Victoria, P.O. Box 3055, Victoria, BC V8W 3P6, Canada

${ }^{5}$ Department of Physics and Astronomy, Aarhus University, Ny Munkegade 120, 8000 Aarhus C, Denmark; kfb@phys.au.dk, fgj@phys.au.dk, bruntt@gmail.com, srf@phys.au.dk

${ }^{6}$ Harvard-Smithsonian Center for Astrophysics, Cambridge, MA 02138; smeibom@cfa.harvard.edu

${ }^{7}$ Center for Interdisciplinary Exploration and Research in Astrophysics (CIERA) and Northwestern University, Department of Physics and Astronomy, 2145 Sheridan Road, Evanston, IL 60208; a-geller@northwestern.edu

${ }^{8}$ Department of Physics and Astronomy, The Johns Hopkins University, Baltimore, MD 21218; imants@pha.jhu . edu

${ }^{9}$ Sydney Institute for Astronomy (SIfA), School of Physics, University of Sydney, NSW, 2006, Australia; stello@physics.usyd.edu.au
} 
to determine a reliable mass for the secondary star and radii for both stars, and to constrain the cluster age. Unlike well-measured stars of similar mass in field binaries, the low-mass secondary is not significantly inflated in radius compared to model predictions. The primary star characteristics, in combination with cluster photometry and masses from other cluster binaries, indicates a best age of $2.62 \pm 0.25 \mathrm{Gyr}$, although stellar model physics may introduce systematic uncertainties at the $\sim 10 \%$ level. We find preliminary evidence that the asteroseismic predictions for red giant masses in this cluster are systematically too high by as much as $8 \%$.

Subject headings: binaries: eclipsing — open clusters and associations: individual (NGC 6819) — stars: low-mass — stars: distances

\section{Introduction}

Long-period eclipsing binary stars are treasures for stellar astrophysicists because they offer the chance to examine the characteristics of (effectively) isolated stars with a high degree of precision and accuracy. Mass and radius in particular can be simultaneously measured to precisions of better than 1\% in many cases (Andersen 1991; Torres et al. 2010). When one or more of the stars in the binary has evolved significantly off of the main sequence, the radius becomes an age-sensitive quantity. And if the binary is a member of a star cluster, this gives us a means of tightly constraining the age of the cluster and testing stellar evolution theory quite strictly (e.g. Brogaard et al. 2012, 2011; Meibom et al. 2009).

Although there have been a number of extensive variability studies of NGC 6819 (Kaluzny \& Shara 1988; Street et al. 2002, 2003, 2005; Talamantes et al. 2010), no eclipsing binary with period greater than about 15 days had been identified in the cluster. This is in part due to a low probability of occurrence, as well as a low probability of detection in studies of short duration. Even though many binary systems have been identified in a long-term radial velocity survey of the cluster (Hole et al. 2009), there had not been a program to look for eclipses among the long-period spectroscopic binaries. The Kepler mission changed this by making continuous photometric monitoring possible, and thanks to a special effort by the science team to study clusters (an effort led by S. Meibom), a large portion of NGC 6819 (as well as the open cluster NGC 6791) is being observed.

\footnotetext{
${ }^{1}$ This is paper $\mathrm{xx}$ of the WIYN Open Cluster Study (WOCS).
} 
Hole et al. (2009) originally discovered WOCS $23009\left(\alpha_{2000}=19^{\mathrm{h}} 41^{\mathrm{m}} 16.848, \delta_{2000}=\right.$ $+40^{\circ} 07^{\prime} 27^{\prime \prime} .55$; also known as KIC 5024447 and A851; Auner 1974) to be a single-lined spectroscopic binary and likely member of the cluster. We first detected the system as an eclipsing binary in quarter 4 of Kepler data, and flagged it as unusual because of the long duration of the eclipse $(\sim 1.8 \mathrm{~d})$. Because the system's photometry places it close to the cluster's main sequence turnoff, the length of the eclipse does not stem solely from a long period, but is also because the radius of the primary star is larger than that of an unevolved main sequence star. A preliminary analysis indicated that the eclipse occurred near apastron, strongly constraining the inclination of the system to be very near $90^{\circ}$, or else the primary eclipse would not have been observed. Although we have only observed three eclipses of the system so far, the rare qualities of this binary make it possible to derive precision measurements of the individual stars already. We therefore present our initial analysis of the stellar characteristics in this paper.

\section{Observational Material and Data Reduction}

\subsection{Spectroscopy}

Spectroscopic observations were obtained as part of the WIYN Open Cluster Study (WOCS; Mathieu 2000). A detailed description of the acquisition and reduction of WOCS observations can be found in Geller et al. (2008), but a summary is presented here. WOCS spectroscopy uses the WIYN 3.5-m telescope 2 on Kitt Peak along with the Hydra multiobject spectrograph $(\mathrm{MOS})$, which is fiber-fed and capable of obtaining $\sim 70$ stellar spectra simultaneously (with $\sim 10$ fibers devoted to sky measurements). The observations presented here used the echelle grating providing a spectral resolution of $\sim 15 \mathrm{~km} \mathrm{~s}^{-1}$. The spectra are centered at $513 \mathrm{~nm}$ with a $25 \mathrm{~nm}$ range in order to cover a rich array of narrow Fe absorption lines near the Mg I b triplet. Spectroscopic observations of our target were completed using 1 hour integrations per visit that were split into three $1200 \mathrm{~s}$ integrations to allow for the rejection of cosmic rays.

Spectroscopic image processing was done within IRAF. After bias and sky subtraction, the extracted spectra were flat-field, throughput, and dispersion corrected. Calibration of the spectra utilized one $200 \mathrm{~s}$ flat field and two bracketing $300 \mathrm{~s}$ ThAr emission lamp spectra. Radial velocities were determined from a one-dimensional cross-correlation with an observed

\footnotetext{
${ }^{2}$ The WIYN Observatory is a joint facility of the University of Wisconsin-Madison, Indiana University, Yale University, and the National Optical Astronomy Observatories
} 
solar spectrum that was corrected to be at rest. The velocities were subsequently converted to the heliocentric frame and corrected for fiber offsets present in the Hydra MOS. The measurement precision is $0.4 \mathrm{~km} \mathrm{~s}^{-1}$ for observations of single-lined systems (such as WOCS 23009) down to a magnitude $V \sim$ 16.5. Hole et al. (2009) presented a summary of NGC 6819 radial velocity measurements, although the present study makes use of more recent spectroscopic observations by the same group. We used a total of 31 observations spanning from June 1998 to September 2010. The phased radial velocity measurements are plotted in Fig. 1, showing the eccentricity of the orbit.

\subsection{Kepler Photometry}

Since quarter 1 as part of the Kepler Cluster Study (Meibom et al. 2011), the Kepler Science Team has recorded a series of 20 large image stamps $(100 \times 20$ pixels $)$ that cover the center of NGC 6819. Together they form a $200 \times 200$ pixel square field of view covering about 13 '25 on a side. The image stamps were taken in long cadence mode, corresponding to exposure times of approximately 30 minutes. Although there are eight quarters of data on NGC 6819 that are publicly available as of this writing, we will discuss quarters 4 (19 December 2009 - 19 March 2010) and 9 (19 March 2011 - 27 June 2011), which contained individual eclipses of WOCS 23009.

We originally detected the primary eclipse in quarter 4 via simple aperture photometry conducted using modified versions of the PyKe $3^{3}$ software tools. For later analysis we made use of the publicly available light curve produced as part of the exoplanet detection program. For quarter 9 observations, a public light curve was not initially available, and so we determined our own light curve using a pixel mask from quarter 5 (corresponding to the same observing season with the star on the same CCD). For our final results, we made use of light curves derived from the Pre-search Data Conditioning (PDC) pipeline (Stumpe et al. 2012; Smith et al. 2012), but we verified that these were good representations of the eclipses by comparing to our own reduction of the raw pixel data. We briefly describe that effort below.

It is well known that the photometer on the Kepler spacecraft introduces significant systematic trends into the photometric data (Kinemuchi et al. 2012). To begin to address this, we applied the kepcotrend algorithm using the first three cotrending basis vectors determined from analysis of light curves on the same CCD. After this procedure, there were still systematic trends visible outside of eclipses. For example, there appears to be spot activity with amplitude comparable to the secondary eclipse, but with period of around 5

\footnotetext{
${ }^{3}$ http://keplergo.arc.nasa.gov/PyKE.shtml
} 
d. We therefore opted to fit low-order polynomials to contiguous sequences of observations (generally separated from each other by momentum desaturation events on the spacecraft), deriving the normalization for the light curves from the fit. (This spot removal was done for PDC light curves as well.)

Before finally normalizing the light curve, we also corrected the flux for crowding. Due to the large pixel size ( $4^{\prime \prime}$ width) and full-width-half-maximum (FWHM) of the point spread functions (95\% encircled energy in a 4 pixel diameter aperture) of stars in the Kepler field, it is difficult to estimate the fluxes from Kepler photometry alone for stars in the crowded cluster fields. However, WOCS 23009 resides in a relatively sparse portion of the cluster, and crowding measures (ratio of target flux to total flux in the optimal photometry aperture) for quarters 4 and 9 (0.919 and 0.928, respectively) from the Kepler Data Search at the Mikulski Archive for Space Telescopes (MAST) (Brown et al. 2011) support the idea that contamination due to other stars is small. We corrected the flux normalization for each quarter of data for this "third light" using the crowding measures above, so that the influence of light from other stars on the Kepler photometry should be negligible. We discuss nearby stellar sources more in \$2.3, The corrected Kepler eclipse light curves are shown in Fig. 2, with the median out-of-eclipse magnitude $\left(m_{K e p, m e d}\right)$ subtracted.

We calculated the center of the Q4 primary eclipse using the method of Kwee \& van Woerden (1956), and found barycentric Julian date (BJD) 2455267.6009 \pm 0.0007 . The long duration of the eclipse (especially ingress and egress) allowed a more precise measurement of mid-eclipse than for most other eclipsing binaries in the Kepler field. Because the Q9 secondary eclipse occurs closer to periastron, the duration was considerably shorter (about $1.1 \mathrm{~d}$ ), and there are fewer observations within the ingress and egress phases. We found BJD 2455695.144 \pm 0.014 .

\subsection{Ground-based Photometry}

In order to more strongly constrain the binary period and obtain color information, we obtained VI images using the Mount Laguna Observatory (MLO) $1 \mathrm{~m}$ telescope on four successive nights (18-21 April 2012) to identify a second occurrence of the primary eclipse. The system was observed to be in eclipse on 20 and 21 April.

The images were processed using standard procedures to apply overscan corrections from each image, to subtract a master bias frame, and to divide a master flat field frame. The photometry was derived using the image subtraction software ISIS (Alard 2000). The general procedure is described in Talamantes et al. (2010), as is some of the older photometric data that were used to help identify the reference out-of-eclipse level. Fig. 2 shows photometry 
from the four nights in April 2012. Although the ground-based images were not taken at the precise bottom of the primary eclipse, the depths of the eclipse in $V$ and $I_{C}$ agree well with the Kepler eclipse depth.

In order to assess the potential contamination of the Kepler photometry from the light of nearby stars, we examined our deepest, best-seeing ground-based image in the $I_{C}$ band. In the vicinity of WOCS 23009, stars had FWHMs of about 3.7 pix or about 1".5. Fig. 3 shows two much fainter stellar sources within $5^{\prime \prime}$ of WOCS 23009. We modeled the point-spread function of the image using DAOPHOT, and found that the two stars are about 3.9 and $5.5 I_{C}$ mag fainter respectively. We also subtracted off WOCS 23009 using ALLSTAR in order to look for closer resolvable stars, but found no additional sources. While these two nearby sources contaminate the light from the binary in the Kepler data, they should have negligible impact on the ground-based photometry using image subtraction techniques.

\section{Analysis}

\subsection{Cluster Membership}

A first important question that must be asked is whether WOCS 23009 is a bona fide member of the cluster. It is not trivial to establish membership for a single star in a cluster, and arguments are generally framed according to probabilities. From an observational standpoint, the strongest membership arguments can be made when there are strong contrasts between the properties of the cluster stars and those of field stars in the same direction. Therefore, we look at several independent membership criteria. First, WOCS 23009 is 4.33 or $\sim 1.8 r_{c}$ from the center of NGC 6819 , where $r_{c}$ is a King model value of the core radius (Kalirai et al. 2001). At this radius, a simple ratio of the spatial density of probable cluster members versus the total spatial density yields a $44 \%$ membership probability. This probability is estimated from Fig. 3 in Kalirai et al. (2001). It should be noted that this membership probability is only a lower limit because no attempt was made to account for the differences in the cluster and field distributions in the color-magnitude diagram (CMD). The CMD position of the binary system mostly reflects the characteristics of the primary star (as shown earlier), and so the fact that the photometry places it among other single cluster members is additional circumstantial evidence of membership.

The cluster membership can be estimated more quantitatively from the radial velocity distribution for an unbiased sample of stars (Hole et al. 2009). Our fit to the binary's system velocity $\gamma$ (see Table 3 ) is very close to the cluster mean radial velocity $\left(2.338 \pm 0.019 \mathrm{~km} \mathrm{~s}^{-1}\right)$ and well within the velocity dispersion $\sigma=1.009 \pm 0.019 \mathrm{~km} \mathrm{~s}^{-1}$. Based on the formulas 
given in Hole et al., we calculate a 93\% membership probability for WOCS 23009. Finally, we have an independent estimate of astrometric cluster membership. Star WOCS 23009 is too faint to be present in the proper-motion study by Sanders (1972) or the asteroseismic membership study of Stello et al. (2011b). We have calculated new proper motions around NGC 6819 down to $V \sim 21$ (Platais et al., in preparation). This study makes use of selected old photographic plates in combination with recent CCD observations at the $3.6 \mathrm{~m}$ CanadaFrance-Hawaii Telescope (CFHT). The accuracy of proper motions for well-measured stars near the center of the cluster is $\sim 0.2$ mas $\mathrm{yr}^{-1}$. For star WOCS 23009, the uncertainty

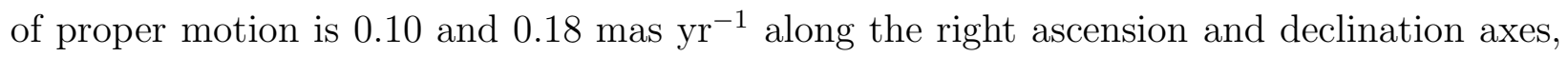
respectively. Images of this star are isolated and optimally exposed on all available plates and CCD mosaic frames. This gives us confidence that the star's astrometry is excellent. The calculated astrometric membership probability for WOCS 23009 is $99 \%$. Summarizing, the body of evidence overwhelmingly supports cluster membership of this star, which should be considered a true cluster member. As such, the properties of the stars contain information on the properties of the cluster.

\subsection{External Constraints on the Binary}

Because WOCS 23009 is a single-lined spectroscopic binary, we needed to place an external constraint on the mass of the primary star to be able to solve for the secondary star mass and both stellar radii. In this respect, the binary's membership in the cluster allows us to utilize external information that is not available for binary stars in the field. Models of the secondary eclipse indicate that the secondary star contributes about $0.19 \%$ of the total system light in the Kepler bandpass, so that out-of-eclipse system photometry can be taken as accurately representing the primary star. Therefore, a comparison to the photometry of other well-measured cluster stars can be used to constrain the primary star's characteristics. We assembled calibrated CCD photometry from available sources (Kalirai et al. 2001; Rosvick \& Vandenberg 1998; Hole et al. 2009; Skrutskie et al. 2006), and we determined shifts from matching stars that would place their photometry on the same magnitude zeropoint as ours in $B, V$, and $I_{C}$ bands. (See Talamantes et al. 2010 for the comparison with Rosvick \& Vandenberg 1998, and Jeffries et al. 2012 for comparisons with Kalirai et al. 2001 and Hole et al. 2009.) Magnitude offsets were never larger than 0.066 mag. The results are shown in Table 1. For the filters where comparison is possible, the measurements are consistent within 0.03 mag.

Though the stars in other NGC 6819 eclipsing binaries do not bracket the characteristics

of the primary in WOCS 23009, they are close enough to allow us to make a modest extrap- 
olation to estimate the mass of its primary star. Jeffries et al. (2012) analyzed ground-based photometry and spectroscopy for the eclipsing binary system WOCS 40007 (also known as A259; Auner 1974), and Table 2 summarizes the results of that study, while Figure 4 shows the deconvolved CMD positions of its stars. The secondary star in the WOCS 40007 system undergoes a total eclipse, so we can accurately measure the difference in $V$ magnitude between the components of the binary and use this as a consistency check on our calculations. The observed value $(\Delta V=0.740)$ agrees with the theoretical predictions for the observed masses of the WOCS 40007 stars.

By forcing isochrones to fit the photometry of the two WOCS 40007 stars at the appropriate masses, we can estimate the primary star's mass for WOCS 23009 using the $V$ magnitude of the primary. We opted to rely more on the isochrone fits to the secondary star of WOCS 40007 because it is closer in mass $\left(M_{s}=1.086 \pm 0.018 M_{\odot}\right)$ to the Sun, so that its interior structure should be understood somewhat better. In addition, because WOCS 40007 has total eclipses of the secondary star and there is a faint tertiary on a long period orbit, the photometry of the secondary star is more tightly dictated (Jeffries et al. 2012). If the primary star of WOCS 40007 had been used to pin down the isochrones, the mass of the WOCS 23009 primary would be systematically larger. However, our quoted uncertainty on the WOCS 23009 primary mass encompasses fits that would match the WOCS 40007 primary. (In other words, the photometry for both stars falls within the ranges predicted by the same isochrone for the measured masses and 1 sigma uncertainties.)

With this algorithm, the theoretical estimates of the mass are relatively insensitive to the exact age of the isochrone used as long as the isochrones approximately match the morphology of the cluster turnoff. The evolution of the star's radius picks up dramatically for stars that have evolved past the reddest point of the kink in the isochrone (see $\$ 4.2$ ). If the mass of any such stars are measured in the future, that will provide a strong upper limit to the mass of the primary of WOCS 23009.

The reader should note that this is a differential estimate of the star mass, and so will avoid many of the potential errors involved in fits to the CMD (even when distance modulus and reddening are somehow constrained). Realistic systematic errors of 0.1 dex in the absolute metallicity scale only change the estimated mass by about $0.01 M_{\odot}$, and potential helium abundance errors probably have a similarly small effect — to first order, composition errors should affect all of the cluster stars to approximately the same degree. Also, while we do base our mass determination on isochrones that fit the turnoff region of the CMD the best, we are not assuming a cluster age at this stage. Depending on the physics in the isochrones used (see Figs. 4and 5), the age could be different, but the observational constraints from the CMD restrict the allowed values of the primary mass. The estimates produced by different 
sets of isochrones agree well, with Dartmouth (Dotter et al. 2008) isochrones producing the lowest mass, Padova (Girardi et al. 2000) and Yonsei-Yale (Demarque et al. 2004) isochrones predicting a value about $0.005 M_{\odot}$ higher, and Victoria-Regina (VandenBerg et al. 2006) and BaSTI (Pietrinferni et al. 2004) isochrones the highest by about $0.015 M_{\odot}$. We have chosen the Dartmouth value as our preferred value because of the overall fit of those isochrones to the turnoff region and the inclusion of significant physics updates (see \$4.2).

The biggest source of uncertainty in the primary mass estimate comes from the masses of the WOCS 40007 stars because they allow for a small range of acceptable alternative fits. For a single set of isochrones, fits that return masses falling within the $1 \sigma$ uncertainties on both of the WOCS 40007 components produce a full range for acceptable fits of about $0.046 M_{\odot}$. Therefore, we estimate $M_{1}=1.468 \pm 0.030 M_{\odot}$ using the midpoint and somewhat conservative $1 \sigma$ error bars (slightly more than half of the full range). We emphasize that the precision and accuracy of the primary star mass estimate will improve as the data on WOCS 40007 improves and as additional binaries in the cluster are used to further constrain the analysis.

Surface temperature is somewhat important for constraining the limb darkening coefficients to be used in the modeling, but is even more important for the distance modulus determination in $\$ 4.2$. Using the system photometry and the empirical color-temperature relations of Casagrande et al. (2010), we can estimate the temperature of the primary from the various optical and infrared colors. We corrected for an average reddening $E(B-V)=0.12 \pm 0.01$ (Jeffries et al. 2012) after transforming this to other colors using the York Extinction Solver 4 (McCall 2004). Because we did not calibrate $R_{C}$ in our photometry, we cannot be certain it has the same zeropoint, and therefore, we give less weight to colors involving that filter. In addition, we give more weight to colors with a combination of small uncertainty in the photometry and small uncertainty on the reddening (when translated from uncertainty in $B-V)$. Based on this, our best estimate of the primary star temperature is $T_{1}=6320 \pm 150$ $\mathrm{K}$.

\subsection{Binary Star Modeling}

To simultaneously model the ground-based radial velocities and photometry and Kepler photometry, we used the ELC code (Orosz \& Hauschildt 2000). As discussed later, we fitted the data in two different ways. In one, we used PHOENIX model atmospheres to describe the limb darkening of the stars and the variation of emitted intensity with emergent

\footnotetext{
${ }^{4}$ http://www2.cadc-ccda.hia-iha.nrc-cnrc.gc.ca/community/YorkExtinctionSolver/
} 
angle. In that case, we fitted the binary with a set of 10 parameters: orbital period $P$, time of conjunction (primary eclipse) $t_{c}$, velocity semi-amplitude of the primary star $K_{p}$, system radial velocity $\gamma$, eccentricity $e$, argument of periastron $\omega$, inclination $i$, ratio of the primary radius to average orbital separation $R_{1} / a$, ratio of radii $R_{2} / R_{1}$, and temperature ratio $T_{2} / T_{1}$. In the second set of trial runs, we fit for one additional parameter: one of the two coefficients in the quadratic limb darkening law for the primary star in the Kepler filter band. Because of the poor time resolution of the secondary eclipse and the faintness of the secondary star, the limb darkening coefficient for the secondary star is poorly constrained and was not fitted.

In our models using the quadratic limb darkening law, one of the two coefficients for the primary star was held fixed at the value given by ATLAS atmospheres (Claret 2000) for the estimated effective temperature and gravity of the star, while the other coefficient was fitted as part of the solution. By fitting for one of the coefficients, the effects of systematic errors in the other can be mitigated because the coefficients tend to be correlated (Southworth et al. 2007). For comparison, we identified the best-fit solution using PHOENIX model atmospheres (Hauschildt et al. 1997). Because the model atmospheres describe the variation of emitted intensity with emergent angle, there is no need to assume limb-darkening coefficients. However, if there are differences between the vertical structure of the atmosphere in the models compared to the stars, this will lead to systematic error in the fitted stellar characteristics (and particularly the radii). Indeed, there are slight mismatches between the observations and best-fit model when using the atmospheres.

The quality of the model fit was quantified by an overall $\chi^{2}$, and the minimum value was sought first using a genetic algorithm (Metcalfe 1999; Charbonneau 1995; Orosz et al. 2002) to explore a large swath of parameter space, followed by Markov chain Monte Carlo modeling (Tegmark et al. 2004) to explore alternate models near the minimum and to estimate the uncertainties in the binary model parameters. The quoted parameter uncertainties are based on the range of values that produce a total $\chi^{2}$ within 1 of the minimum value, which approximates a $1 \sigma$ uncertainty (Avni 1976). The same procedure was used to determine uncertainties in the radii of both stars and the secondary mass. (Uncertainties are not tabulated for the runs utilizing model atmospheres due to the computing time needed to explore the parameter space.)

Because the uncertainties for the measurements are used in the calculation of $\chi^{2}$, it is important these uncertainties are as realistic as possible. We therefore forced the measurement uncertainties to be consistent with the observed scatter around the best fit model. If the measurement uncertainties calculated during the data reduction process are underestimated (as is often the case), this would inflate the total $\chi^{2}$ value and lead to an underestimation of the uncertainties on the binary star model parameters. To be conservative, after initial 
optimization runs for the binary star model, the uncertainty estimates for the radial velocity and photometric observations were scaled upward to return a reduced $\chi^{2}$ value of 1 for each type of measurement. (In other words, the reduced $\chi^{2}$ value for radial velocities was used to scale the radial velocity uncertainties, and so on.) After this scaling (by the square root of the reduced $\chi^{2}$ ), we computed new models to determine the binary model parameters.

Figure 6 shows the results of exploring the multi-dimensional parameter space during the binary star modeling for the fits with a limb-darkening law. The reader should note that the panels show the full range of the parameter space covered by models having $\chi^{2}$ within 5 of the best fit model. More than $10^{6}$ models were run to survey the parameter space. Figs. 1 and 7 show comparisons of the radial velocities and Kepler light curves with the best fit model. Table 3 shows the parameters of the best fits using the limb-darkening law and model atmospheres. Most of the parameters are consistent within the uncertainties. Because the limb-darkening law fit matches the eclipse data more exactly, we will use the measurements from that run in the discussion below.

\section{Discussion}

Because we needed to apply an external constraint on the primary's mass to solve for the secondary star mass and both radii, our solution of the binary is not completely independent of stellar models, and will potentially be subject to systematic errors. Before discussing the results below, we make some estimates of how severe these errors might be.

Given the measurable mass function $\left(f=M_{2}^{3} \sin ^{3} i /\left(M_{1}+M_{2}\right)^{2}\right)$ for this single-lined spectroscopic binary, changes in the choice of primary mass will affect secondary mass determinations like $\partial M_{2} / \partial M_{1}=2 q /(q+3)$ where $q=M_{2} / M_{1}$ is the mass ratio. In other words, the systematic error in the secondary mass will be about $20 \%$ the size of the systematic error in the primary mass for this binary, assuming all other uncertainties are negligible. The masses of both stars influence radius measurements mostly through the total orbital velocity $V=v_{1}+v_{2}$ because timing uncertainties are very small for the Kepler observations of the long eclipses for this system. Even though we do not have measurements of the secondary star velocities or the semi-amplitude $K_{2}$, the effect on the total orbital velocity goes like $\frac{\partial V}{\left(K_{1}+K_{2}\right)} \approx \frac{\partial\left(K_{1}+K_{2}\right)}{\left(K_{1}+K_{2}\right)}=\frac{K_{1}}{\left(K_{1}+K_{2}\right)} \partial\left(1+\frac{M_{1}}{M_{2}}\right)=\frac{1}{q+3} \frac{\partial M_{1}}{M_{1}}$. For the WOCS 23009 binary, this implies that fractional errors in radii will only be about $30 \%$ as large as the fractional systematic error in the primary mass.

So even with a significant systematic error on the primary mass, a good constraint on the secondary mass and radii for both stars can still be obtained. If the primary mass has 
a systematic error of about $0.045 M_{\odot}$, a systematic error of only $1 \%$ in the radii will be introduced.

Because errors in the primary star mass and radius are correlated, the error ellipse in the $M-R$ plane is tilted with respect to the axes (see Fig. 8). This makes the error ellipse somewhat more parallel to the isochrones, potentially reducing the age uncertainty. However, after examining the best fit models (ones with $\chi^{2}$ within 1 of the minimum value), we find the tilt is approximately $25^{\circ}$, which has a minimal effect.

Although systematic errors in $M_{2}$ and $R_{2}$ are both correlated with $M_{1}$ (potentially making the error ellipse more parallel to the model main sequence, as in Fig. 9), we find this effect to be even smaller, producing a tilt of only about $9^{\circ}$. The error bars plotted in Fig. 9 therefore give a very good approximation to the edges of the $1 \sigma$ error ellipse. As a result, it is unlikely that a systematic error in the primary mass is responsible for substantial error in the secondary radius. Regardless, surface gravity should be even less sensitive to potential errors because the correlations will mostly cancel. As illustrated in the lower right panel of Fig. 8, any correlation of $\log g_{2}$ with the primary mass appears to be minimal, meaning that it is likely to be reliable.

These exercises underline the fact that the primary star radius as well as the secondary star mass and radius are relatively insensitive to the primary star mass estimate for the same reasons - that this is a totally-eclipsing binary.

\subsection{The Low-Mass Secondary Star}

Only a handful of field stars are measured to have $M<0.5 M_{\odot}$ with small ( $<3 \%$ ) mass and radius uncertainties, and all of these are in eclipsing binaries. As Fig. 9 shows, the stars in binaries that have been studied generally show radii that are significantly larger than models predict (see the figure caption for references on individual systems, and López-Morales 2007 for a review). The hypotheses that are currently being debated are that the larger radii stem from either a higher level of activity (with convection inhibited by strong magnetic fields or fast rotation) or a higher metallicity. Metallicity is difficult to measure directly for lowmass stars due to the complexity of their spectra, but indications are that a metallicity trend is only evident among single low-mass stars (López-Morales 2007). Coughlin et al. (2011) recently described preliminary evidence from Kepler binaries that spin-up due to a companion in short period binaries is responsible for inflated radii. However, Feiden \& Chabover (2012) have also emphasized that age is an additional uncertainty in comparisons of observations and models because even fewer systems provide age information. 
The WOCS 23009 binary star system has made it possible to precisely measure the characteristics of a low-mass star in the cluster NGC 6819. This is the first such star measured in an open cluster, and as a result of its membership, we have excellent metallicity and age information from other cluster members. The most recent spectroscopic measurements indicate a slightly super-solar composition for NGC 6819 - in the most detailed study to date, Bragaglia et al. (2001) find $[\mathrm{Fe} / \mathrm{H}]=+0.09 \pm 0.03$ from three red clump stars, where the quoted uncertainty is the error in the mean (systematic uncertainties are probably larger). Another unique aspect of this system is its large orbital period (a factor of 10 larger than any other known low-mass eclipsing binary), which allows the secondary star to evolve like it is in virtual isolation.

As seen in Fig. 9, our measurements indicate that the secondary star is one of the few well-measured low-mass stars with a radius that is consistent with model predictions. The models of Baraffe et al. (1998) pass slightly outside our conservative mass uncertainty, although the agreement would improve if those models used a chemical composition consistent with the super-solar metallicity measured for NGC 6819. Our measurements are completely consistent with more recent models (Dotter et al. 2008, validated in calculations by Feiden et al. 2011) that use an updated equation of state for the stellar gas.

The closest observational comparison, CU Cnc A (Ribas 2003), has approximately the same radius $\left(R=0.4317 \pm 0.0052 R_{\odot}\right)$ and lower surface gravity $(\log g=4.804 \pm 0.011)$ for a slightly smaller mass $\left(M=0.4333 \pm 0.0017 M_{\odot}\right)$. If CU Cnc A is a member of the Castor moving group (Ribas 2003), this would provide a metallicity $([\mathrm{Fe} / \mathrm{H}]=0.0)$ and age estimate (320 \pm 80 Myr; Ribas 2003). Then CU Cnc A and WOCS 23009 B would provide a test of the idea that metallicity is the cause of larger-than-predicted radii. Before that is a strong test though, the mass uncertainty on WOCS 23009 B needs to be reduced, and CU Cnc A's membership in the moving group needs to be verified (see Feiden \& Chabover 2012 for a critique).

The number of systems having low-mass stars and both reliable age and metallicity information is very limited. Feiden \& Chaboyer (2012) identify just 3 binaries with reliable information of this type, and all three have short periods that probably produce problems with stellar activity effects. Two (KOI-126 and CM Dra) contain stars near $0.2 M_{\odot}$, while the other (YY Gem) contains stars near $0.6 M_{\odot}$. While the CM Dra and YY Gem systems have stars with larger-than-predicted radii, the two low-mass stars in KOI-126 have radii consistent with models for an age of $4 \pm 1$ Gyr, like that of the evolved third star in the system.

WOCS 23009 B therefore gives us a very interesting opportunity: the opportunity to test models with a low-mass star that has well-determined and precise mass, radius, age, 
and composition. The star has a radius and gravity that appear consistent with model predictions, and it is more compact than its closest observational comparison, CU Cnc $\mathrm{A}$, at the $1.4 \sigma$ level. The long period of the binary assures us that tidal interactions are very unlikely to induce activity in the secondary star, unlike the situation for $\mathrm{CU}$ Cnc A $(P=2.771 \mathrm{~d})$. In contrast, the low-mass eclipsing binaries with the previous records for the largest orbital periods (about $41 \mathrm{~d}$ ) are LSPM J1112+7626 A and B (Irwin et al. 2011) and Kepler 16B (Dovle et al. 2011); both are found to be significantly larger than models predict, but do not have age or (in the case of LSPM J112+7626) metallicity constraints that allow us to test alternate hypotheses.

Low levels of intrinsic stellar activity could be partially responsible for the small radius of WOCS 23009 B, but the activity level of the star will be difficult to characterize because of the large difference in luminosities between the components of the binary. Although spot activity can affect radius measurements in eclipsing binaries, spot activity on the lowmass secondary in this system should be a minimal influence because the secondary star contributes such a small proportion of the system light. Based on the mass and likely age of the primary star, spots probably affect the photometry minimally. However, future eclipse observations with Kepler will allow us to monitor the system for unexpected changes accompanying the star's activity cycle.

\subsection{The Cluster Age and Distance Modulus}

We first examine the mass-radius diagram as this is the most direct way of comparing the observations to models. Radii of evolved stars can make good age indicators because they can be measured to high precision in eclipsing binary systems and they generally avoid sources of systematic errors (like distances and reddening) that can influence other age determinations. The biggest concern for age determination using the WOCS 23009 eclipsing binary is the reliability of the primary star mass estimate. As we emphasized in \$3.3, the primary star mass estimate $i s$ effectively constrained by a mass-luminosity relationship for main sequence (or near main sequence) stars. In contrast to the situation for the secondary star, there is a significant correlation between the primary star mass and radius. However, we evaluated this effect at the beginning of the Discussion section, and we use an appropriately tilted error ellipse in our analysis.

To make the connection between stellar radius and age, theoretical models are necessary, and systematic differences in the input physics will affect the inferred age. While it is possible to find good fits to the primary star of WOCS 23009 when the isochrones are forced to match the components of WOCS 40007 in the CMD (as in Figs. 4 and 5), the ages implied by 
these fits show significant differences, indicating that there are important disagreements in the physics inputs. Table 4 summarizes some of the more important assumptions (from our point of view) in presently available model sets. In addition to those considerations, there has been an important nearly 50\% downward revision to the rate for the CNO cycle bottleneck reaction ${ }^{14} \mathrm{~N}(p, \gamma){ }^{15} \mathrm{O}$ (Marta et al. 2008). Because the strength of the CNO cycle strongly affects the extent of convection in stars with small central convection zones, this probably has a significant effect on inferred ages for this cluster. None of the current publicly available isochrone sets includes this rate revision with the exception of the Dartmouth isochrones, which use an earlier value (Imbriani et al. 2004) that is within 10\% of the Marta et al. value. Due to the inclusion of this reaction rate as well as helium and heavy-element diffusion, we have used the Dartmouth isochrones as our primary theoretical comparison. Our future work will use additional stars in other eclipsing binaries in NGC 6819 and will employ isochrones with updated physics from various theoretical groups.

Fig. 10 shows the comparison in the $M-R$ plane. For the purposes of illustration, we have used identical ages for all of the theoretical isochrones, and when available, the same $[\mathrm{Fe} / \mathrm{H}]$. The precision due to uncertainty in the measurements of the primary star of WOCS 23009 is approximately $0.25 \mathrm{Gyr}$, but the systematic differences between isochrone sets (due to differences in coded physics) are similar to (if not larger than) this. Based on the Dartmouth set, the preferred age is about 2.65 Gyr. This is consistent to within the measurement uncertainties of the age implied by the lower-mass stars in the binary WOCS 40007, and previous isochrone fits to the CMD (2.5 Gyr, Kalirai et al. 2001; 2.4 Gyr, Rosvick \& Vandenberg 1998). The Victoria-Regina, Padova, and BaSTI isochrones imply younger ages (with the Padova and BaSTI results lower by more than 0.25 Gyr or 10\%).

As we have emphasized, even though the primary star mass is somewhat uncertain, this affects the radius measurement in a minor way, and so it is still possible (in concert with a $T_{\text {eff }}$ estimate) to compute the luminosity of the star. Along with a bolometric correction, the absolute magnitude and apparent distance modulus can be calculated. We calculated the bolometric correction in $V$ from VandenBerg \& Clem (2003), and used it along with the model radius and photometric temperature estimate from $\$ 3.3$ to find $M_{V}=2.70$ and $(m-M)_{V}=12.39 \pm 0.10$. The largest contributor to the uncertainty in this distance modulus by far is the temperature.

Using the same method for calculating the distance modulus of WOCS 23009 A (including the employment of photometric $T_{\text {eff }}$ estimates $)$, we determine $(m-M)_{V}=12.36 \pm 0.10$ and $12.41 \pm 0.11$ for the two components of WOCS 40007. The distance moduli from the three stars agree within the uncertainties, and the averaged distance modulus from the binary star components $\left[(m-M)_{V}=12.39 \pm 0.06\right]$ is slightly larger than previous determinations 
$(12.30 \pm 0.10$ from red clump stars, Jeffries et al. 2012; 12.35 from fits to older isochrones, Rosvick \& Vandenberg 1998), but consistent within the errors. The use of a consistent temperature scale for all three stars will tend to minimize scatter in the distance modulus values, although systematic error in the distance moduli will still be present if there is a systematic error in the temperature scale. The most likely cause of such an error is the reddening, although metallicity error could also contribute. Because of their systematic nature, we add the contribution from the reddening $\left(\sigma_{E(B-V)}=0.02\right.$ produces about $70 \mathrm{~K}$ uncertainty in temperature and $0.05 \mathrm{mag}$ in distance modulus) and metallicity $\left(\sigma_{[\mathrm{Fe} / \mathrm{H}]}=0.10\right.$ dex produces $30 \mathrm{~K}$ uncertainty in temperature and 0.02 in distance modulus) in quadrature with the error in the mean for the three stars $(0.06 \mathrm{mag})$ to get our final estimate $(m-M)_{V}=12.39 \pm 0.08$.

We remind the reader that we chose the lowest (Dartmouth) estimate of the primary star mass to use in our binary star analysis. If the mass was systematically higher, this would result in a slightly larger radius (due to correlated errors), a larger calculated luminosity, and thus a larger distance modulus. So the mass estimate is unlikely to be responsible for our larger measurement.

Any comparison in the CMD using just the distance modulus and reddening to shift the theoretical isochrones will immediately be subject to issues relating to color- $T_{\text {eff }}$ transformations, but we do a preliminary comparison here. Again, we use Dartmouth isochrones as our primary theoretical choice, here because they (and the Victoria-Regina set) employ the VandenBerg \& Clem (2003) transformations. VandenBerg et al. (2010) found that those relations show good agreement with the empirical relations from Casagrande et al. (2010) and with recent MARCS atmosphere models. In the $(V, B-V)$ CMD of Fig. 11, the Dartmouth models provide a good fit to the binary components and to single stars near the turnoff. The models appear to be slightly offset to the blue relative to the cluster stars, but this shift can be accounted for by the uncertainties in the reddening and distance, and in color-temperature transformations. The age indicated by the CMD position of the primary star of WOCS 23009 is consistent with the determination from the $M-R$ plane.

An examination of Figs. 4 and 10 shows that the WOCS 23009 primary star is at an interesting point in its evolution, shortly before its radius begins to change rapidly. This evolutionary acceleration occurs shortly after the red kink in the CMD isochrones when convection in the core of the star starts to shut off shortly before central hydrogen exhaustion. Comparing models of a given age with and without convective core overshoot (e.g., Pietrinferni et al. 2004), the radius of a star is unaffected by core overshooting until it has evolved past the red kink. Because the primary star has not reached the cluster's red kink at $(V, B-V) \approx(14.8,0.65)$, the age determined from the stellar masses and radii are not sensitive to the amount of convective overshooting. The morphology of the turnoff and sub- 
giant branch in the CMD are sensitive to overshooting, however. So if we use the binary star results in concert with the CMD, we can put new restrictions on the amount of overshoot. Larger amounts of convective overshooting bring more fuel to the fusion region of the core, and allows more massive stars to reach greater ages and higher luminosities before they turn onto the subgiant branch. When comparing to the Dartmouth models, the subgiant branch appears to be about $0.1 \mathrm{mag}$ too faint, so this might be addressed with a larger amount of convective core overshoot. While the amount of overshoot in the Dartmouth models is similar to what is used in most other isochrone sets (see Table 4 for a comparison of the overshoot in units of pressure scale height $H_{P}$ ), the lack of a match to the subgiant branch may be related to the lower (but more recent) ${ }^{14} \mathrm{~N}(p, \gamma){ }^{15} \mathrm{O}$ reaction rate used in the Dartmouth models. Because this reaction sets the rate of energy generation in the CNO cycle and because the CNO cycle is responsible for convective cores in these main sequence stars, the revised reaction rate probably will affect stellar models in a manner similar to reduced convective overshooting. Further work combining analysis of the eclipsing binaries and CMD will be important in addressing this physics issue, which affects the masses of stars reaching the giant branch.

The constraints that can be placed on the turnoff mass are also important to the asteroseismic work on red giant stars in this cluster (Stello et al. 2010, 2011a, b; ;iglio et al. 2012; Corsaro et al. 2012). Asteroseismology using the observed quantities $\Delta \nu$ (the frequency separation of overtone modes) and $\nu_{\max }$ (the frequency of maximum power) holds great promise for deriving the characteristics of isolated stars, but model-independent validation of the resulting radii and masses is still underway (in eclipsing red giants, for example; Hekker et al. 2010). The so-called direct method uses $\Delta \nu$ and $\nu_{\max }$ (along with $T_{\text {eff }}$ ) to compute mass and radius from scaling relations. Examination of the scaling relation linking $\Delta \nu$ and the mean density $\left(\Delta \nu / \Delta \nu_{\odot} \simeq \sqrt{\rho / \rho_{\odot}}\right)$ using stellar models shows that the relation probably holds to the level of a few percent for a wide range of evolutionary states (Stello et al. 2009; White et al. 2011). The scaling relation involving $\nu_{\max }$ is thought to relate to the atmospheric acoustic cutoff frequency (see Mosser et al. 2010 for some empirical validation), which in turn relates to surface gravity and effective temperature $\left(\nu_{\max } / \nu_{\max , \odot} \simeq\left(g / g_{\odot}\right) / \sqrt{T_{\text {eff }} / T_{\text {eff, } \odot}}\right.$; Brown et al. 1991). When tested against stellar models, this relation also appears to hold within a few percent (Stello et al. 2009). When these two relations are combined, the radius and mass of a star can be calculated if $T_{\text {eff }}$ is known, although the asteroseismic observables almost entirely cancel each other in the expression for mass due to the correlation between them (Stello et al. 2009). "Grid-based" methods (finding best fits to $\Delta \nu, \nu_{\max }, T_{\text {eff }}$, and $[\mathrm{Fe} / \mathrm{H}]$ values in a grid of models) avoid some of these problems, but it is still found that masses are less precisely determined than radii and subject to greater systematic errors (Gai et al. 2011). While asteroseismic radii can be tested using techniques like interferom- 
etry (Huber et al. 2012), validation of asteroseismic masses is still at an early stage due to a lack of calibration stars with independent mass determinations. (See Kallinger et al. 2010 for an example of a calibration using a heterogeneous sample of red giants.)

Because Kepler asteroseismic results have been used to derive masses for NGC 6819 giants, a comparison with our eclipsing binary masses can be made. Two Kepler asteroseismic results on stellar masses in NGC 6819 have been reported: Basu et al. (2011) found an average giant mass of $1.68 \pm 0.03 M_{\odot}$ using a grid-based method, while Miglio et al. (2012) found a consistent value of $1.61 \pm 0.03 M_{\odot}$ purely using 4 different asteroseismic scaling relations involving various combinations of $\Delta \nu, \nu_{\max }, L$, and $T_{\text {eff }}$. For comparison, the bestfitting Dartmouth isochrone predicts that the mass at the bluest point on the isochrone (at the blue end of the subgiant branch) is about $1.52 M_{\odot}$ and the mass of stars at the tip of the giant branch is about $1.56 M_{\odot}$. When any of the sets of isochrones are forced to fit the components of WOCS 40007 (as in Figs. 4 and 5), the red giant masses are lower than the Basu et al. asteroseismic value by between about 0.07 (Yonsei-Yale, Padova) and $0.13 M_{\odot}$ (BaSTI). While the BaSTI result is probably affected by the lower amount of overshoot used in their models, there does not seem to be a set of models that simultaneously matches the masses and radii of the eclipsing binary components, the morphology of the turnoff in the CMD, and the Basu et al. asteroseismic mass determination for red giants.

Although the Miglio et al. result is consistent with some models (Padova, VictoriaRegina, and Yonsei-Yale) to within the uncertainties, it is still slightly discrepant with the preferred Dartmouth models. In addition, three of the four asteroseismic scaling relations used by Miglio et al. involve luminosity, and so require a distance modulus. The distance modulus used by them is smaller than we find for the eclipsing binaries by about 0.18 mag. Adoption of the binary star distance modulus would result in more luminous stars and larger asteroseismic mass estimates, so this would worsen the disagreement. In the same vein, the distance modulus for NGC 6819 derived by Basu et al. $\left[(m-M)_{0}=11.85 \pm 0.05\right]$ is also smaller than implied by the binary stars.

At this point, our results are indicative of a systematic error in the asteroseismic masses, although it must be remembered that this ultimately rests on the masses derived from the WOCS 40007 system and the radius of the primary star in WOCS 23009. Additional binary systems in the cluster are currently being analyzed and their measurements will improve our ability to predict turnoff and giant masses. The constraints from eclipsing binaries in this cluster and others in the Kepler field will help provide a more solid basis for asteroseismic determinations of mass if disagreements like these can be resolved. 


\section{Conclusions}

We present the discovery of total eclipses in a long period (771.8 d) eccentric binary system that is a member of the open cluster NGC 6819. The primary star is somewhat evolved and significantly larger than a main sequence star of the same mass, allowing us to derive an age of $2.62 \pm 0.25$ Gyr for the cluster using published models with the most up-to-date physics inputs. From the three cluster stars in eclipsing binaries that have been measured so far, we find an apparent distance modulus $(m-M)_{V}=12.39 \pm 0.08$, where the uncertainty comes from the weighted mean and systematic uncertainties in reddening and metallicity.

The measurement precision for the characteristics of this binary star (with the possible exception of the star masses) will improve with additional spectroscopic and photometric eclipse observations. Two of the largest uncertainties in the current analysis involve the stellar temperatures and the primary star mass. Better determination of the stellar surface temperatures will significantly improve the distance modulus determination, and a more precise determination of the reddening (via Stromgren photometry, for example) would allow a consistency check using colors. The primary mass will be more difficult to address, but new isochrone sets with improved physics inputs [such as the ${ }^{14} \mathrm{~N}(p, \gamma){ }^{15} \mathrm{O}$ reaction rate, diffusion, and convective core overshooting] and flexible chemical composition selection will help improve the estimate made here. However, it should be remembered that a solution for this binary system would not have been possible without constraints provided by other members of the cluster. Even with the relatively large uncertainty on the primary star mass (about $2 \%$ ), we are able to reach a precision on radius measurements of better than $1 \%$ for both stars. In turn, this allows us to examine other stellar astrophysics questions.

Even with the difficulties introduced by the large luminosity contrast between the two stars, the cluster age precision is determined to better than $10 \%$. The characteristics of the stars we have studied so far are not sensitive to core convective overshooting, but the morphology of the turnoff in the color-magnitude diagram is, and this fact should make NGC 6819 an excellent calibrator for the theoretical description of overshooting.

The secondary star in the binary falls in the low-mass regime at about $0.45 M_{\odot}$, but it does not show signs of radius inflation, as most other stars in the mass range do. Although high metallicity has been postulated as a cause of the larger radii, the secondary star contradicts this - NGC 6819's metallicity is super-solar $([\mathrm{Fe} / \mathrm{H}]=+0.09)$. While the secondary star in WOCS 23009 has a seemingly normal radius and it now holds the record for the low-mass star in the longest period eclipsing binary, two other relatively long-period systems $(P \approx 41 \mathrm{~d})$ contain stars that do show inflated radii. Episodic stellar activity may help explain these observations, and monitoring of the radii of stars in this and other long-period 
binaries would help address whether this affects the measurements in an unexpected way. Further reduction of the mass uncertainty for the secondary will make this star a stronger test of low-mass stellar models.

Finally, we emphasize that the mining of the ensemble of information available for this cluster has just begun. Although our results indicate that there is still some disagreement between masses derived from binaries and those derived from asteroseismology of giants in this cluster, the joint use of both techniques shows the potential for improving stellar modeling by pointing out where our understanding needs improvement. With additional constraints on the mass of the primary star, whether they come from asteroseismology or other eclipsing binaries, the age determination for NGC 6819 is likely to improve as the result of additional observations from the ground and with Kepler.

We are very grateful to the Kepler team for the opportunity to work with such a precise and extensive dataset for detecting variable stars. We would also like to thank G. Feiden for helpful conversations. Our work has been funded through grant AST 09-08536 from the National Science Foundation and grant NNX11AC76G from the National Aeronautics and Space Administration to E.L.S. K.B. acknowledges support from the Carlsberg Foundation. The WIYN Open Cluster Study has most recently been supported by National Science Foundation grant AST-0908082.

This paper includes data collected by the Kepler mission. Funding for the Kepler mission is provided by the NASA Science Mission directorate. This research made use of the SIMBAD database, operated at CDS, Strasbourg, France; the NASA/ IPAC Infrared Science Archive, which is operated by the Jet Propulsion Laboratory, California Institute of Technology, under contract with the National Aeronautics and Space Administration; the WEBDA database, operated at the Institute for Astronomy of the University of Vienna; and the Mikulski Archive for Space Telescopes (MAST). STScI is operated by the Association of Universities for Research in Astronomy, Inc., under NASA contract NAS5-26555. Support for MAST is provided by the NASA Office of Space Science via grant NNX09AF08G and by other grants and contracts. 


\section{REFERENCES}

Andersen, J. 1991, A\&A Rev., 3, 91

Alard, C. 2000, A\&AS, 144, 363

Asplund, M., Grevesse, N., Sauval, A. J., \& Scott, P. 2009, ARA\&A, 47, 481

Auner, G. 1974, A\&AS, 13, 143

Avni, Y. 1976, ApJ, 210, 642

Baraffe, I., Chabrier, G., Allard, F., \& Hauschildt, P. H. 1998, A\&A, 337, 403

Basu, S., et al. 2011, ApJ, 729, L10

Bragaglia, A. et al. 2001, ApJ, 121, 327

Brogaard, K., Bruntt, H., Grundahl, F., et al. 2011, A\&A, 525, A2

Brogaard, K., VandenBerg, D. A., Bruntt, H., et al. 2012, A\&A, 543, A106

Brown, T. M., Gilliland, R. L., Noyes, R. W., \& Ramsey, L. W. 1991, ApJ, 368, 599

Brown, T. M., Latham, D. W., Everett, M. E., \& Esquerdo, G. A. 2011, AJ, 142, 112

Carter, J. A., Fabrycky, D. C., Ragozzine, D., et al. 2011, Science, 331, 562

Casagrande, L., Ramírez, I., Meléndez, M., \& Asplund, M. 2010, A\&A, 512, A54

Casagrande, L., Portinari, L., \& Flynn, C. 2006, MNRAS, 373, 13

Charbonneau, P. 1995, ApJS, 101, 309

Coughlin, J. L., López-Morales, M., Harrison, T. E., Ule, N., \& Hoffman, D. I. 2011, AJ, 141,78

Claret, A. 2000, A\&A, 363, 1081

Corsaro, E., Stello, D., Huber, D., et al. 2012, arXiv:1205.4023

Demarque, P., Woo, J.-H., Kim, Y.-C., \& Yi, S. K. 2004, ApJS, 155, 667

Dotter, A. et al. 2008, ApJS, 178, 89

Doyle, L. R., Carter, J. A., Fabrycky, D. C., et al. 2011, Science, 333, 1602 
Feiden, G. A., Chaboyer, B., \& Dotter, A. 2011, ApJ, 740, L25

Feiden, G. A., \& Chaboyer, B. 2012, arXiv:1207.3090

Gai, N., Basu, S., Chaplin, W. J., \& Elsworth, Y. 2011, ApJ, 730, 63

Geller, A. G., Mathieu, R. D., Harris, H. C., \& McClure, R. D. 2008, AJ, 135, 2264

Girardi, L., Bressan, A., Bertelli, G., \& Chiosi, C. 2000, A\&AS, 141, 371

Grevesse, N., \& Noels, A. 1993, Origin and Evolution of the Elements, 15

Grevesse, N., \& Sauval, A. J. 1998, Space Sci. Rev., 85, 161

Hauschildt, P. H., Baron, E., \& Allard, F. 1997, ApJ, 483, 390

Hekker, S., Debosscher, J., Huber, D., et al. 2010, ApJ, 713, L187

Hole, K. T., Geller, A. M., Mathieu, R. D., Platais, I., Meibom, S., \& Latham, D. W. 2009, AJ, 138, 159

Huber, D. et al. 2012, submitted

Imbriani, G., Costantini, H., Formicola, A., et al. 2004, A\&A, 420, 625

Irwin, J. M., Quinn, S. N., Berta, Z. K., et al. 2011, ApJ, 742, 123

Jeffries, M. W. et al. 2012, in preparation.

Kallinger, T., Weiss, W. W., Barban, C., et al. 2010, A\&A, 509, A77

Kalirai, J. S. et al. 2001, AJ, 122, 266

Kaluzny, J., \& Shara, M. M. 1988, AJ, 95, 785

Kinemuchi, K., Barclay, T., Fanelli, M., et al. 2012, arXiv:1207.3093

Kwee, K. K., \& van Woerden, H. 1956, Bull. Astron. Inst. Netherlands, 12, 327

Lopez-Morales, M., Orosz, J. A., Shaw, J. S., et al. 2006, arXiv:astro-ph/0610225

López-Morales, M. 2007, ApJ, 660, 732

Marta, M., Formicola, A., Gyürky, G., et al. 2008, Phys. Rev. C, 78, 022802

Mathieu, R. D. 2000, Stellar Clusters and Associations: Convection, Rotation, and Dynamos, 198,517 
McCall, M. L. 2004, AJ, 128, 2144

Meibom, S., Grundahl, F., Clausen, J. V., et al. 2009, AJ, 137, 5086

Meibom, S., Barnes, S. A., Latham, D. W., et al. 2011, ApJ, 733, L9

Metcalfe, T. S., Mathieu, R. D., Latham, D. W., \& Torres, G. 1996, ApJ, 456, 356

Metcalfe, T. S. 1999, AJ, 117, 2503

Miglio, A., Brogaard, K., Stello, D., et al. 2012, MNRAS, 419, 2077

Mosser, B., Belkacem, K., Goupil, M.-J., et al. 2010, A\&A, 517, A22

Orosz, J. A., Groot, P. J., van der Klis, M., et al. 2002, ApJ, 568, 845

Orosz, J. A., \& Hauschildt, P. H. 2000, A\&A, 364, 265

Peña, J. H., Fox Machado, L., García, H., et al. 2011, Rev. Mexicana Astron. Astrofis., 47, 309

Pietrinferni, A., Cassisi, S., Salaris, M., \& Castelli, F. 2004, AJ, 612, 168

Ribas, I. 2003, A\&A, 398, 239

Ribas, I. 2003, A\&A, 400, 297

Rosvick, J. M., \& Vandenberg, D. A. 1998, AJ, 115, 1516

Sanders, W. L. 1972, A\&A, 19, 155

Skrutskie, M. F. et al. 2006, AJ, 131, 1163

Smith, J. C., Stumpe, M. C., Van Cleve, J. E., et al. 2012, arXiv:1203.1383

Southworth, J., Bruntt, H., \& Buzasi, D. L. 2007, A\&A, 467, 1215

Stello, D., Chaplin, W. J., Basu, S., Elsworth, Y., \& Bedding, T. R. 2009, MNRAS, 400, L80

Stello, D., Basu, S., Bruntt, H., et al. 2010, ApJ, 713, L182

Stello, D., Huber, D., Kallinger, T., et al. 2011, ApJ, 737, L10

Stello, D., Meibom, S., Gilliland, R. L., et al. 2011, ApJ, 739, 13

Street, R. A., et al. 2002, MNRAS, 330, 737 
Street, R. A., et al. 2003, MNRAS, 340, 1287

Street, R. A., et al. 2005, MNRAS, 358, 795

Stumpe, M. C., Smith, J. C., Van Cleve, J. E., et al. 2012, arXiv:1203.1382

Talamantes, A., Sandquist, E. L., Clem, J. L., Robb, R. M., Balam, D. D., \& Shetrone, M. 2010, AJ, 140, 1268

Tegmark, M., Strauss, M. A., Blanton, M. R., et al. 2004, Phys. Rev. D, 69, 103501

Torres, G., Andersen, J., \& Giménez, A. 2010, A\&A Rev., 18, 67

VandenBerg, D. A., Bergbusch, P. A., \& Dowler, P. D. 2006, ApJS, 162, 375

VandenBerg, D. A., Casagrande, L., \& Stetson, P. B. 2010, AJ, 140, 1020

VandenBerg, D. A., \& Clem, J. L. 2003, AJ, 126, 778

White, T. R., Bedding, T. R., Stello, D., et al. 2011, ApJ, 743, 161

Woo, J.-H., Gallart, C., Demarque, P., Yi, S., \& Zoccali, M. 2003, AJ, 125, 754 


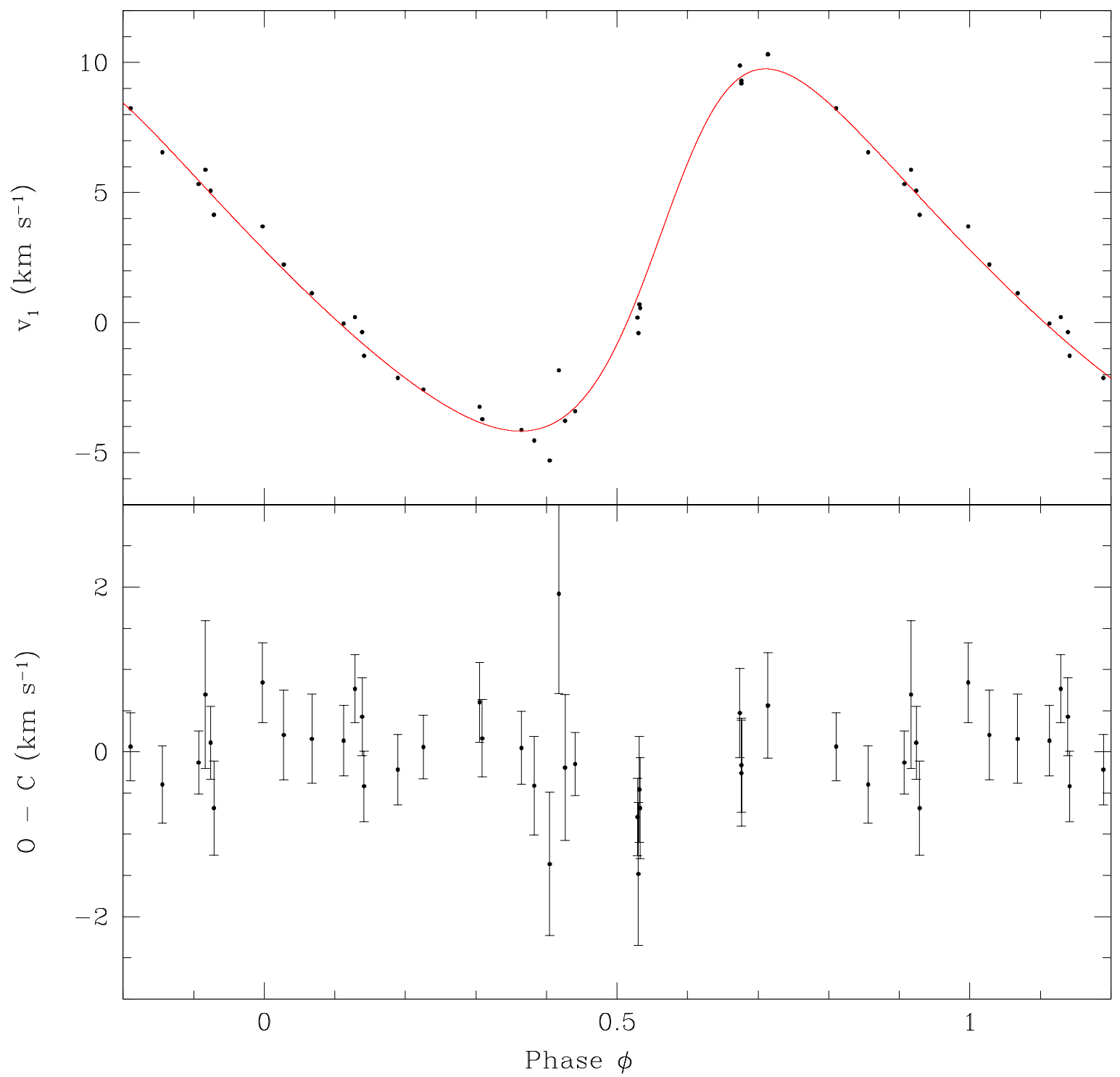

Fig. 1.- Phased WOCS radial velocities for WOCS 23009, along with the best fit limbdarkening law model (red line). The lower panel shows the observed minus computed values with error bars scaled to give a reduced $\chi^{2}=1$ (see $\$ 3.3$ ). 


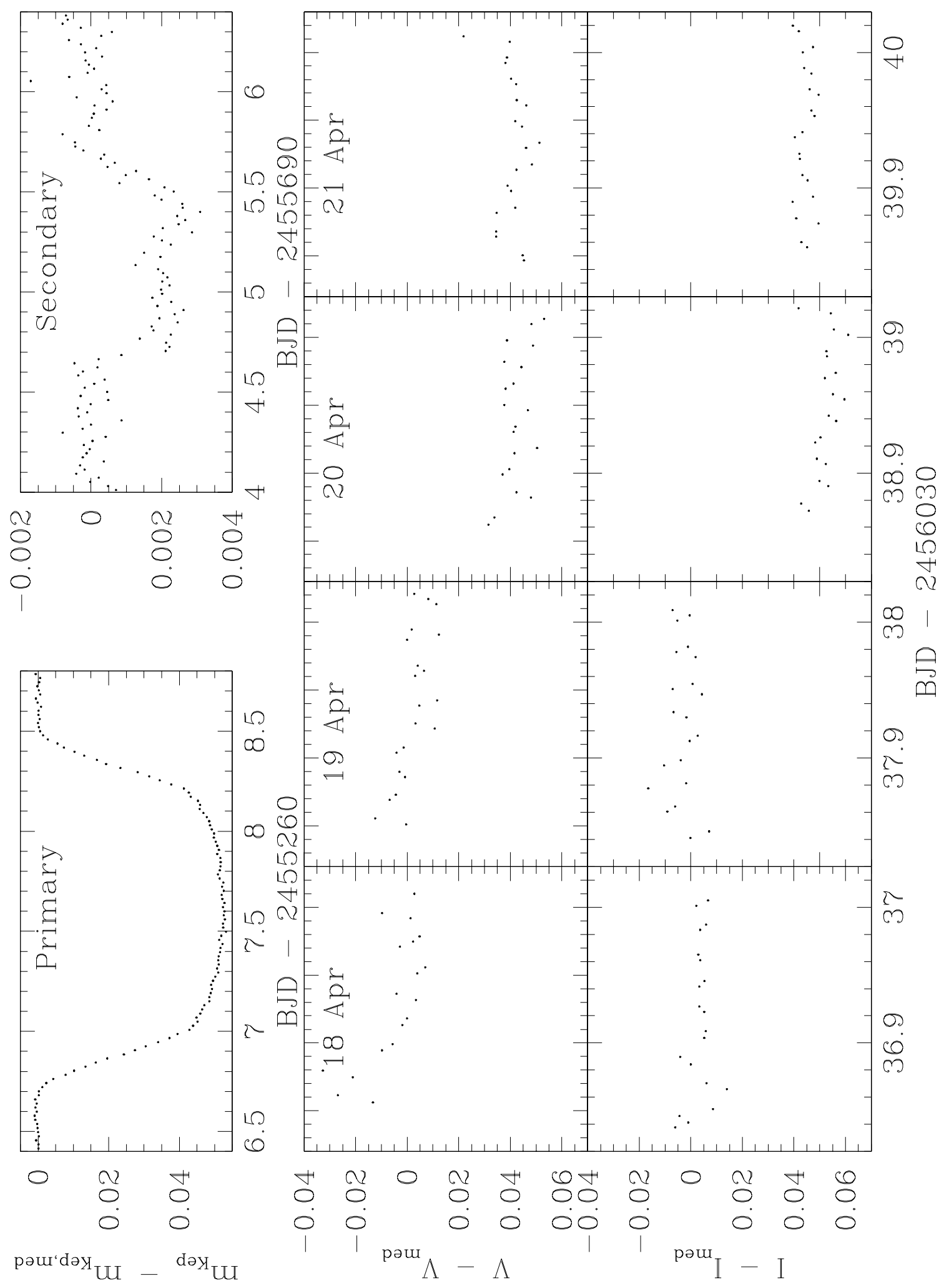

Fig. 2.- Top row: Kepler photometry of the primary and secondary eclipse of WOCS 23009. Bottom rows: Ground-based $V I_{C}$ photometry of the primary eclipse from April 2012. 


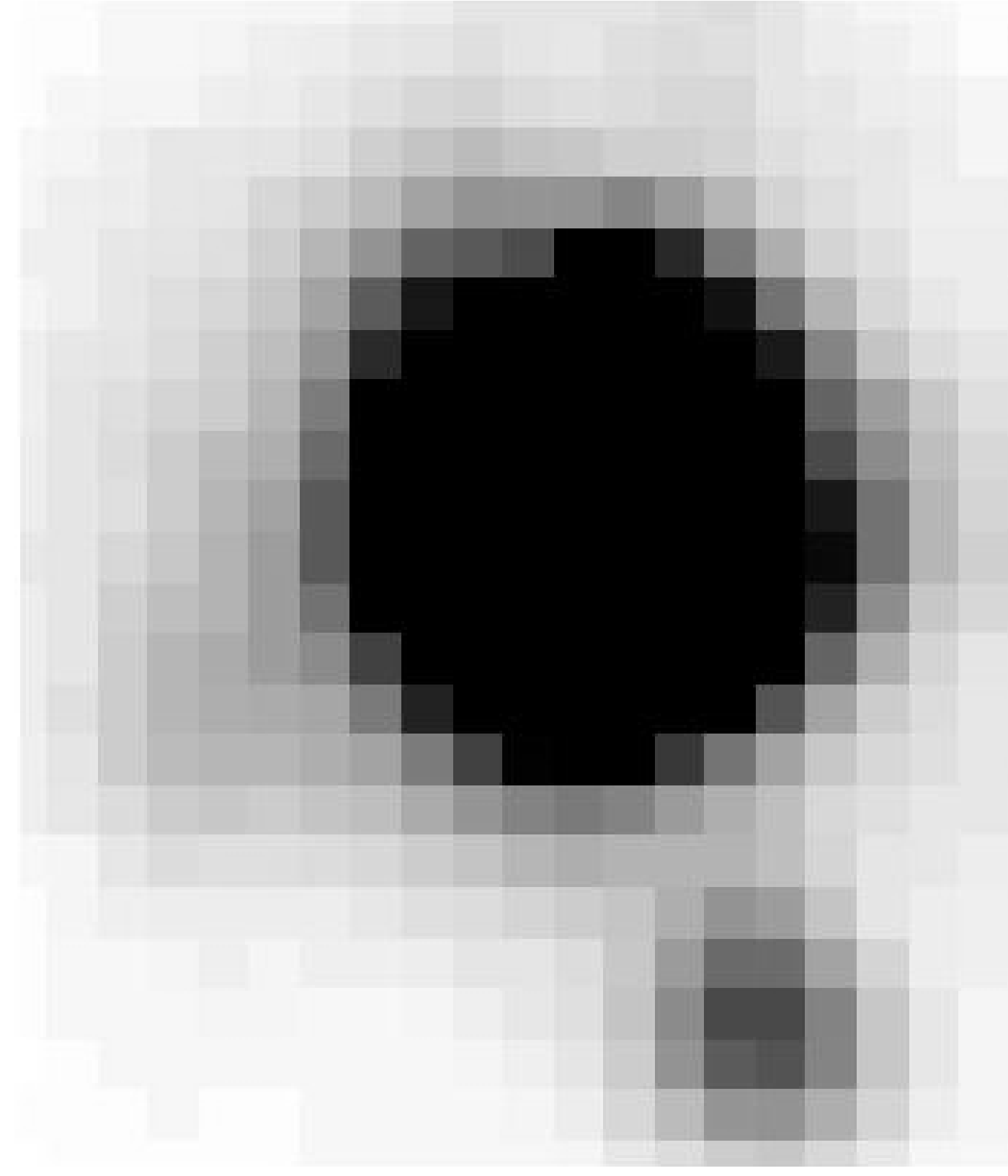

Fig. 3.- An examination of a $10^{\prime \prime}$ section of a ground-based $I_{C}$ image to look for objects that could contaminate photometric measurements of WOCS 23009 (center). Detected stars are at 5 o'clock and 8 o'clock relative to the binary. Each pixel is approximately 0 " 4 on a side. $\mathrm{N}$ is up and $\mathrm{E}$ is to the left. 


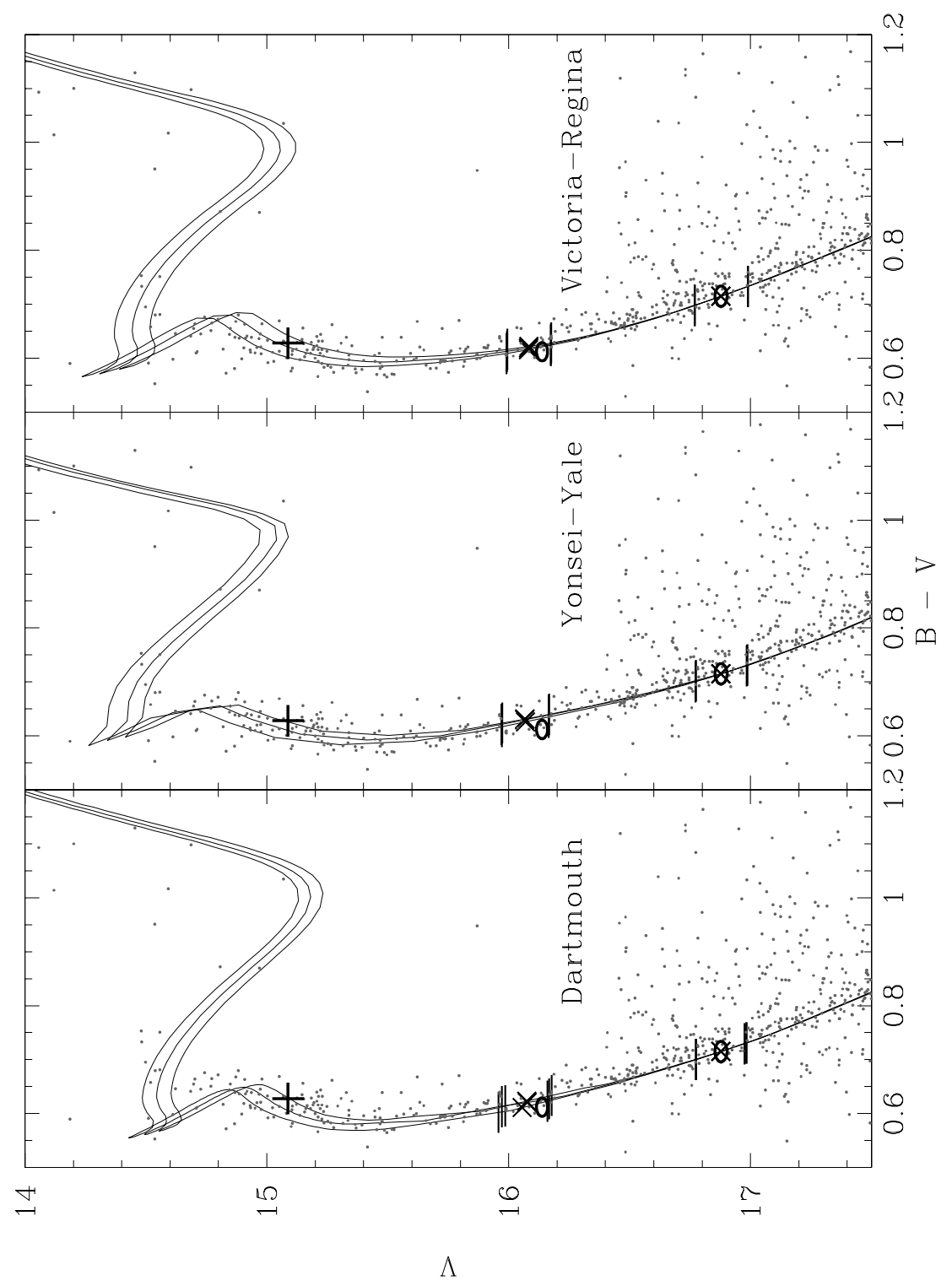

Fig. 4.- Color-magnitude diagram for NGC 6819 (data from Kalirai et al. 2001) with probable single-star cluster members selected for $V \lesssim 16.5$ using Hole et al. (2009). The system photometry for WOCS 23009 (dominated by the primary star) is shown with + , and the deconvolved photometry of the eclipsing stars in WOCS 40007 are shown with ellipses. Isochrones are from the Dartmouth (Dotter et al. 2008; $[\mathrm{Fe} / \mathrm{H}]=+0.09 ; 2.5,2.6,2.7 \mathrm{Gyr}$ ), Yonsei-Yale (Demarque et al. 2004; $[\mathrm{Fe} / \mathrm{H}]=+0.09 ; 2.4,2.5,2.6 \mathrm{Gyr}$ ), and Victoria-Regina (VandenBerg et al. 2006; $[\mathrm{Fe} / \mathrm{H}]=+0.136$; ages 2.4, 2.5, $2.6 \mathrm{Gyr}$ ) groups. The theoretical predictions for stars with masses equal to the WOCS 40007 stars are shown with $\times$, and the uncertainties in mass are delineated above and below those points. 


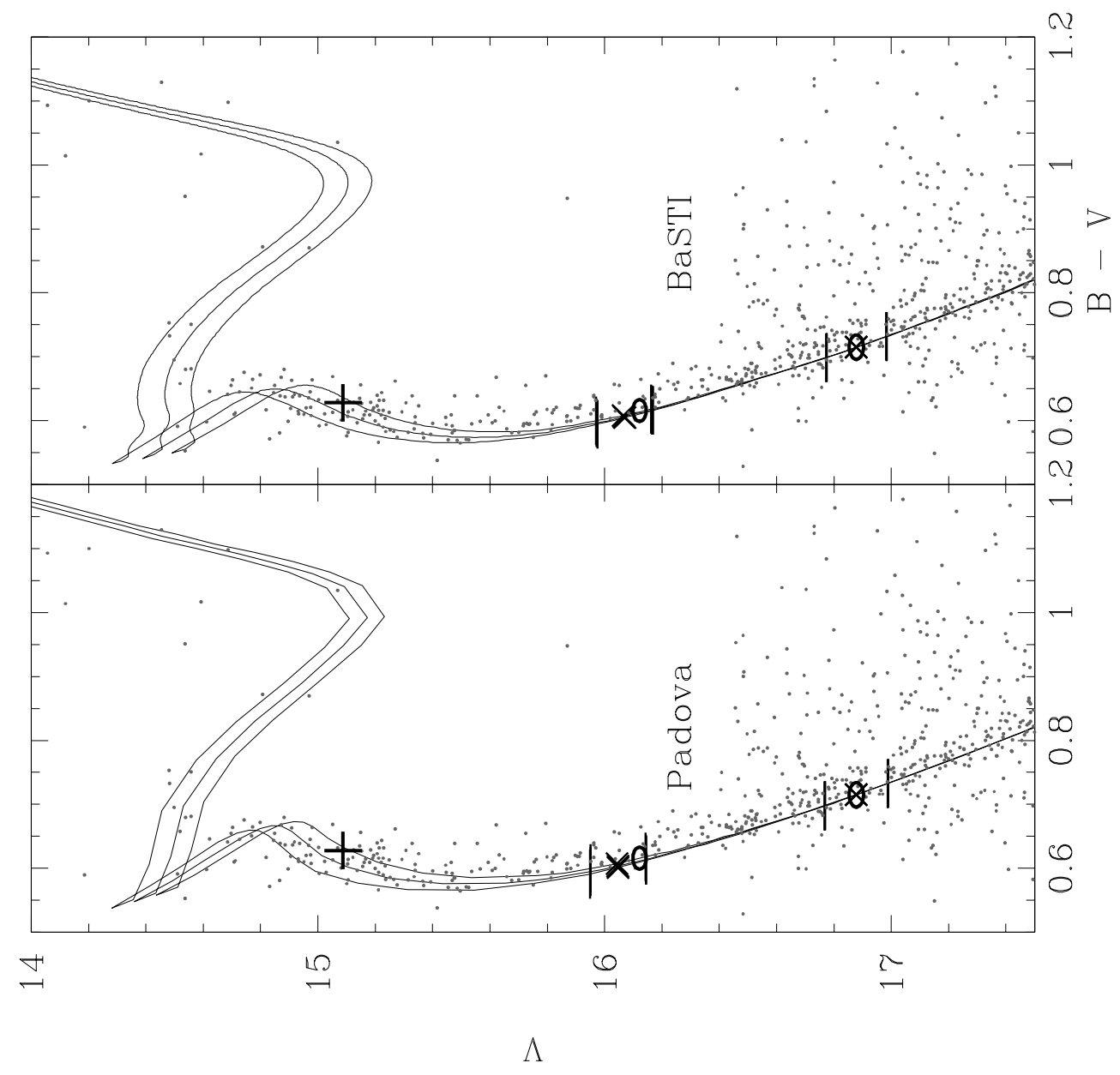

Fig. 5.- Same as for Fig. (4 except for Padova (Girardi et al. 2000; $[\mathrm{Fe} / \mathrm{H}]=+0.09 ; 2.2,2.3$, $2.4 \mathrm{Gyr}$ ), and BaSTI (Pietrinferni et al. 2004; $[\mathrm{Fe} / \mathrm{H}]=+0.06 ; 2.1,2.2,2.3 \mathrm{Gyr}$ ) isochrones. 

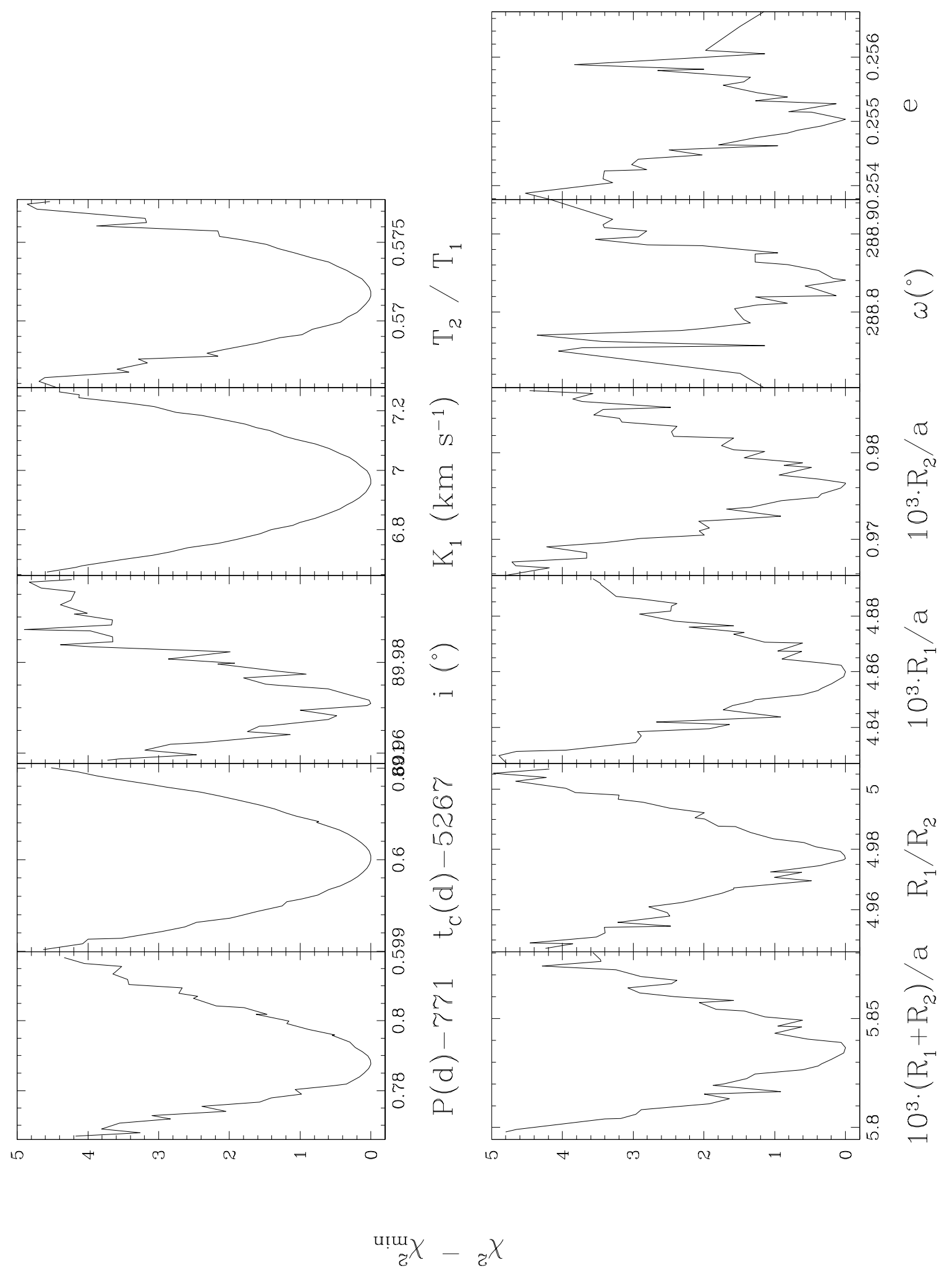

Fig. 6. $-\chi^{2}$ values for best-fit models using a limb darkening law as a function of binary model fitting parameters. 

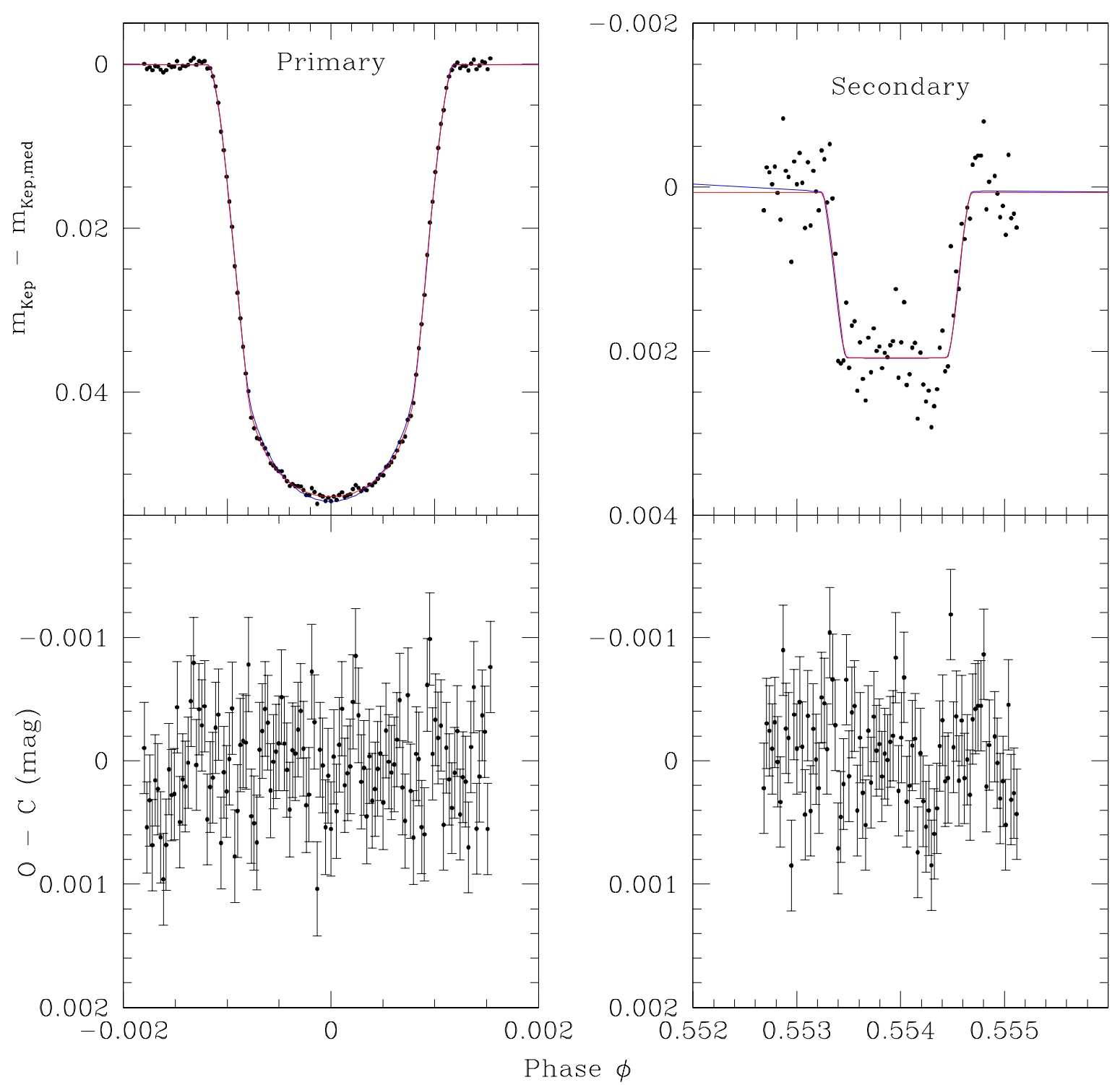

Fig. 7.- Top row: Comparison of Kepler photometry of the eclipses with model fits using a limb-darkening law (red line) and PHOENIX model atmospheres (blue line). Bottom row: Observed magnitudes minus model predictions for the limb-darkening solution with error bars scaled to give reduced $\chi^{2}=1$. 

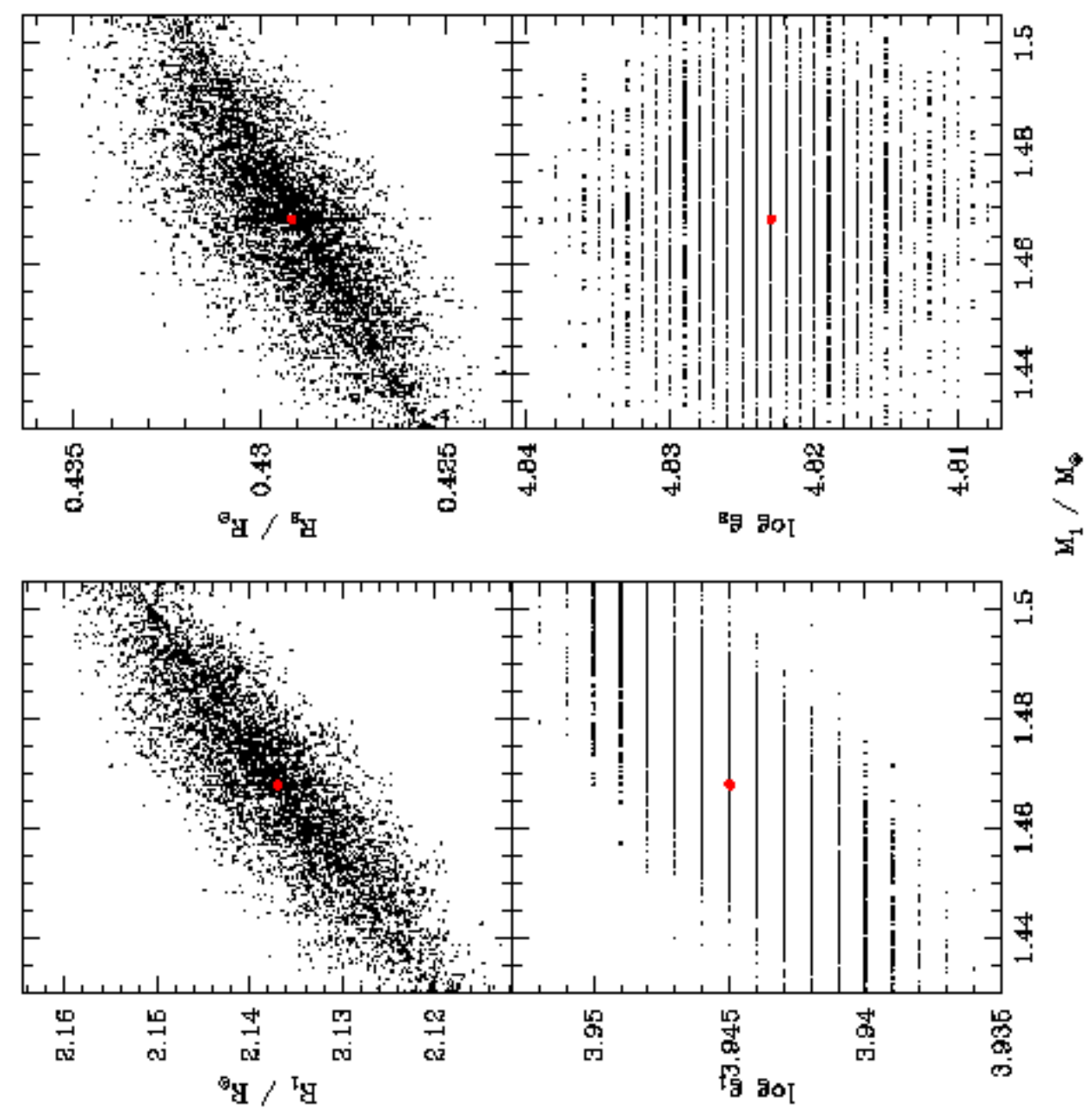

Fig. 8.- Limb-darkened models with primary mass within $1 \sigma$ of the value extrapolated from WOCS 40007 components, and total $\chi^{2}$ within 4 of the best fit model. 


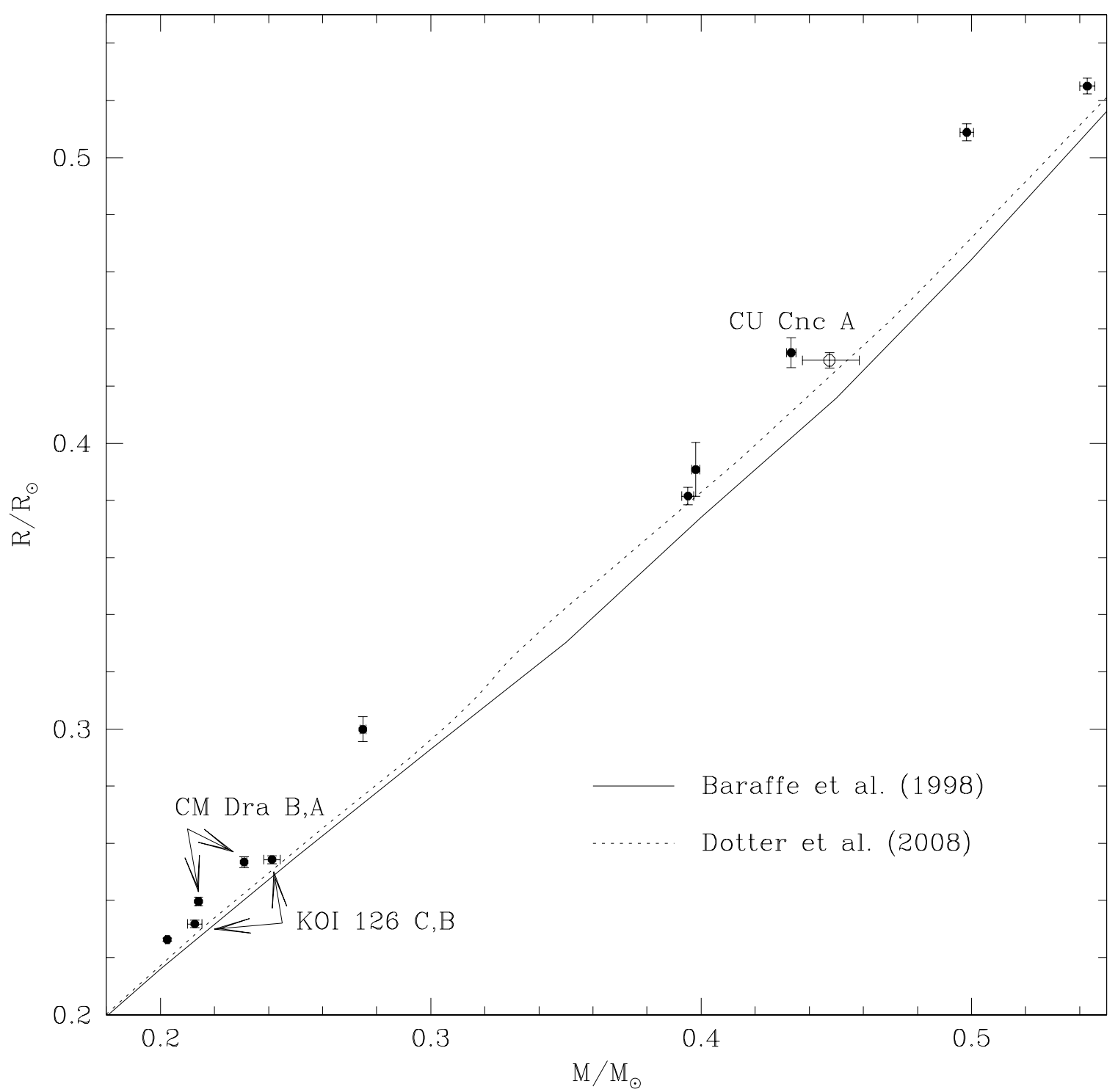

Fig. 9.- Mass-radius plot for low-mass members of eclipsing binary systems having measurement uncertainties of less than 3\%. The secondary star in WOCS 23009 is shown with an open circle, and measurements of other systems were taken from the following references: KOI126 B and C, Carter et al. (2011); CM Dra A and B, Metcalfe et al. (1996); CU Cnc A and B, Ribas (2003); LSPM J1112+7626 A and B, Irwin et al. (2011); Kepler 16B, Doyle et al. (2011); and NSVS01031772 A and B, Lopez-Morales et al. (2006). Model values are shown for $[\mathrm{Fe} / \mathrm{H}]=0$ and age 2.5 Gyr from Baraffe et al. (1998), and $[\mathrm{Fe} / \mathrm{H}]=+0.09$ and age 2.6 Gyr from Dotter et al. (2008). 


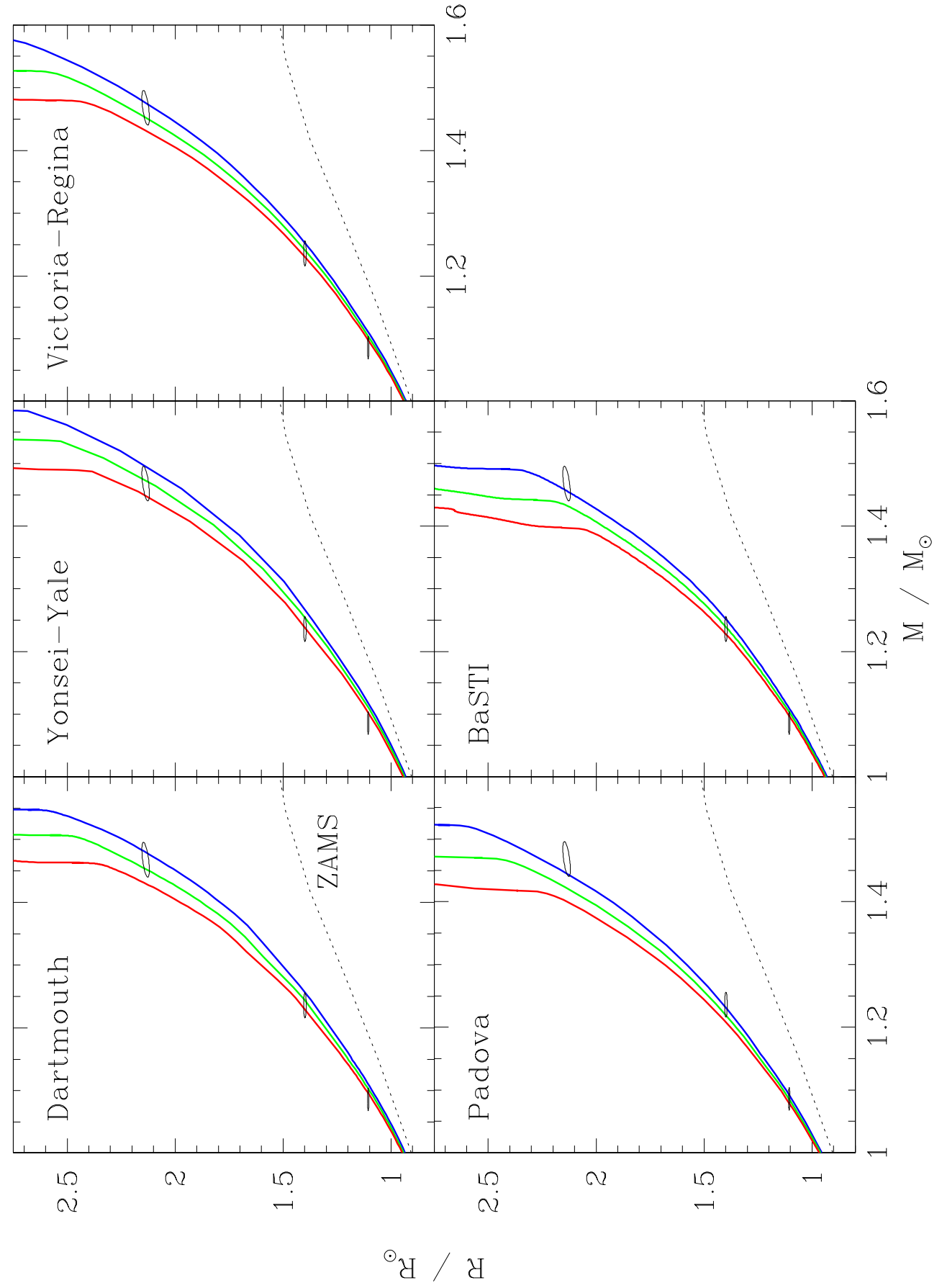

Fig. 10.- Mass-radius plot for members of eclipsing binary systems near the cluster turnoff. The error ellipse for the primary star in WOCS 23009 has the highest mass, and measurements of WOCS 40007 are from Jeffries et al. (2012). Models have ages of 2.5, 2.75, and 3.0 Gyr (from bottom to top). The Dartmouth, Yonsei-Yale, and Padova isochrones use [Fe/H] $=+0.09$, while the Victoria-Regina set uses the nearest tabulated $[\mathrm{Fe} / \mathrm{H}]=+0.136$, and the BaSTI set uses $[\mathrm{Fe} / \mathrm{H}]=+0.06$. 


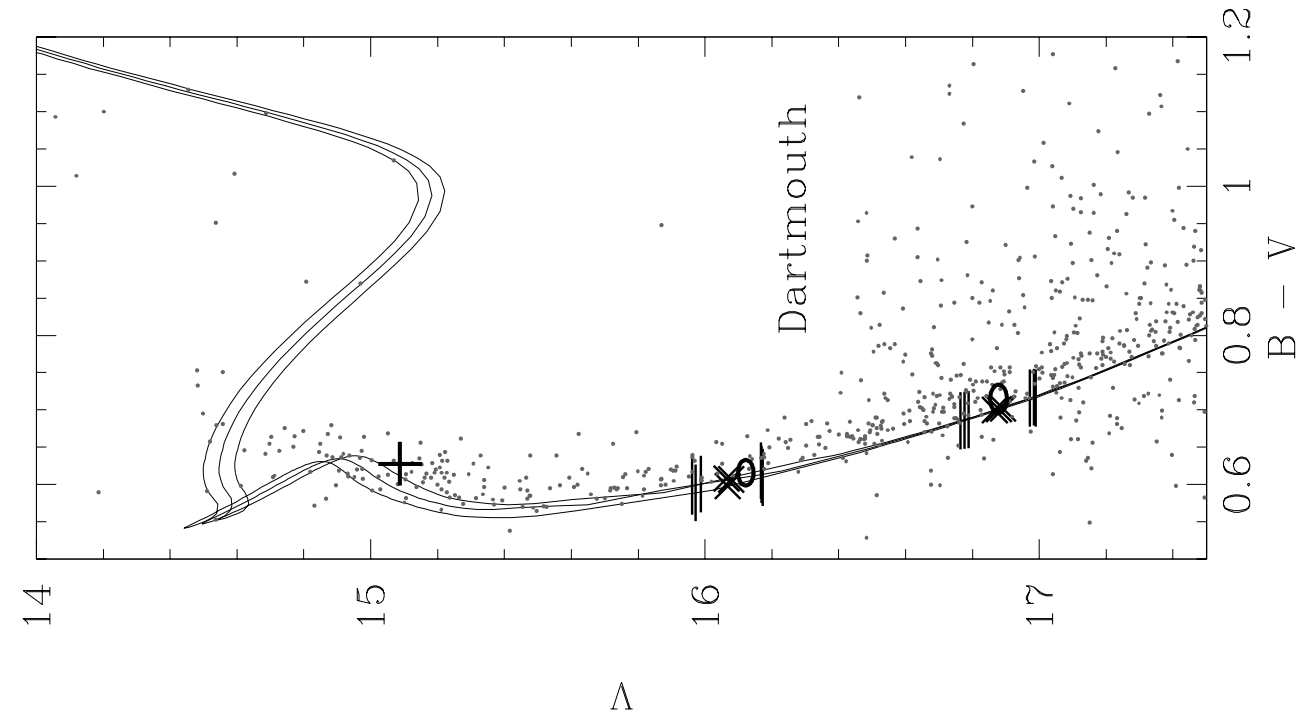

Fig. 11. - Same as Fig. 4 except that isochrones are shifted according to the preferred $E(B-V)=0.12$ and $(m-M)_{V}=12.39$. Isochrones are from the Dartmouth group (Dotter et al. 2008; $[\mathrm{Fe} / \mathrm{H}]=+0.09 ; 2.5,2.6,2.7 \mathrm{Gyr}$ ), and ages were chosen to produce agreement with the bright component of WOCS 23009 (marked by + ). 
Table 1. Out-of-Eclipse Photometry for WOCS 23009 (A851)

\begin{tabular}{lcccc}
\hline \hline Filter & T10/2MASS & K01 & RV98 & H09 \\
\hline$B$ & $15.714 \pm 0.010$ & 15.716 & 15.706 & 15.710 \\
$V$ & $15.090 \pm 0.010$ & 15.088 & 15.104 & 15.098 \\
$R_{C}$ & & & 14.735 & \\
$I_{C}$ & $14.327 \pm 0.009$ & & 14.356 & 14.331 \\
$J$ & $13.834 \pm 0.023$ & & & \\
$H$ & $13.584 \pm 0.027$ & & & \\
$K_{S}$ & $13.556 \pm 0.045$ & & & \\
\hline
\end{tabular}

Note. - T10: Talamantes et al. (2010). 2MASS: Skrutskie et al. (2006). K01: Kalirai et al. (2001). RV98: Rosvick \& Vandenberg (1998). H09: Hole et al. (2009).

Table 2. Summary Data for WOCS 40007 (A259) Binary Components (Jeffries et al. 2012)

\begin{tabular}{lcc}
\hline \hline & Primary & Secondary \\
\hline$M / M_{\odot}$ & $1.236 \pm 0.020$ & $1.086 \pm 0.018$ \\
$R / R_{\odot}$ & $1.399 \pm 0.007$ & $1.098 \pm 0.004$ \\
$V$ & 16.138 & 16.878 \\
$(B-V)$ & 0.612 & 0.715 \\
$T_{\text {eff }}(\mathrm{K})^{\mathrm{a}}$ & $6250 \pm 150$ & $5870 \pm 150$ \\
\hline
\end{tabular}

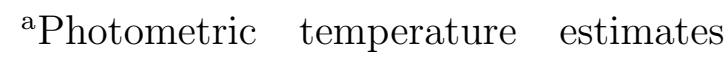
(Casagrande et al. 2010). 
Table 3. Best-Fit Model Parameters for WOCS 23009 (A851)

\begin{tabular}{|c|c|c|}
\hline Parameter & Limb Darkening Law & Atmospheres \\
\hline$M_{1} / M_{\odot}$ & \multirow{2}{*}{\multicolumn{2}{|c|}{$\begin{array}{c}1.468 \pm 0.030 \text { (constraint) } \\
6320 \pm 150 \text { (constraint) }\end{array}$}} \\
\hline$T_{1}(\mathrm{~K})$ & & \\
\hline$x_{K, 1}$ & \multicolumn{2}{|c|}{0.3187 (fixed) } \\
\hline$y_{K, 1}$ & \multicolumn{2}{|l|}{$0.31 \pm 0.02$} \\
\hline$x_{K, 2}$ & \multicolumn{2}{|l|}{0.4013 (fixed) } \\
\hline$x_{V, 1}$ & \multicolumn{2}{|l|}{0.3419 (fixed) } \\
\hline$y_{V, 1}$ & \multicolumn{2}{|l|}{0.3507 (fixed) } \\
\hline$x_{I, 1}$ & \multicolumn{2}{|l|}{0.1800 (fixed) } \\
\hline$y_{I, 1}$ & \multicolumn{2}{|l|}{0.3596 (fixed) } \\
\hline$\gamma\left(\mathrm{km} \mathrm{s}^{-1}\right)$ & $2.22 \pm 0.02$ & 2.21 \\
\hline$P(\mathrm{~d})$ & $771.788_{-0.009}^{+0.010}$ & 771.790 \\
\hline$t_{C}$ & $2455267.6000 \pm 0.0004$ & 2455267.6000 \\
\hline$i\left(^{\circ}\right)$ & $89.971_{-0.004}^{+0.006}$ & 89.974 \\
\hline$e$ & $0.2550 \pm 0.0004$ & 0.2551 \\
\hline$\omega\left(^{\circ}\right)$ & $288.84 \pm 0.04$ & 288.84 \\
\hline$K_{P}\left(\mathrm{~km} \mathrm{~s}^{-1}\right)$ & $6.96 \pm 0.13$ & 6.96 \\
\hline$R_{1} / a$ & $0.004860 \pm 0.000016$ & 0.004899 \\
\hline$R_{2} / a$ & $0.0009765_{-0.0000025}^{+0.0000038}$ & 0.0009634 \\
\hline$R_{2} / R_{1}$ & $4.977_{-0.009}^{+0.006}$ & 5.085 \\
\hline$\left(R_{1}+R_{2}\right) / a$ & $0.005836 \pm 0.000020$ & 0.005863 \\
\hline$T_{2} / T_{1}$ & $0.572 \pm 0.003$ & 0.569 \\
\hline$M_{2} / M_{\odot}$ & $0.447_{-0.011}^{+0.010}$ & 0.446 \\
\hline$R_{1} / R_{\odot}$ & $2.136 \pm 0.014$ & 2.151 \\
\hline$R_{2} / R_{\odot}$ & $0.4292 \pm 0.0033$ & 0.4229 \\
\hline $\log g_{1}(\mathrm{cgs})$ & $3.945 \pm 0.004$ & 3.937 \\
\hline $\log g_{2}(\operatorname{cgs})$ & $4.823 \pm 0.009$ & 4.834 \\
\hline
\end{tabular}


Table 4. Summary of Relevant Inputs for Model Isochrone Sets

\begin{tabular}{lrrlllr}
\hline \hline \multicolumn{1}{c}{ Isochrone } & & \multicolumn{4}{c}{ Diffusion? } & Overshoot \\
& $(Z / X)_{\odot}{ }^{\mathrm{a}}$ & $\Delta Y / \Delta Z$ & $Y$ & $Z$ & $\lambda_{O V} / H_{P}$ \\
\hline BaSTI & 0.0280 & 1.4 & $\mathrm{~N}^{\mathrm{b}}$ & $\mathrm{N}^{\mathrm{b}}$ & 0.14 \\
Dartmouth & 0.0267 & 1.54 & $\mathrm{Y}$ & $\mathrm{Y}$ & 0.20 \\
Padova & 0.0268 & 2.25 & $\mathrm{~N}$ & $\mathrm{~N}$ & $\sim 0.23$ \\
Victoria-Regina & 0.0268 & 2.2 & $\mathrm{~N}$ & $\mathrm{~N}$ & $\mathrm{c}$ \\
Yale-Yonsei & 0.0253 & 2.0 & $\mathrm{Y}$ & $\mathrm{N}$ & 0.20 \\
\hline
\end{tabular}

${ }^{a}$ Initial values are tabulated if diffusion is incorporated.

${ }^{\mathrm{b}}$ Only the solar calibration for the BaSTI models incorporates diffusion of helium $(Y)$ and heavy elements $(Z)$.

${ }^{\mathrm{c}}$ The Victoria-Regina models use a different algorithm for convective core overshooting, so it is difficult to directly compare to the other sets. 